\title{
Hyperkähler cones and instantons on quaternionic Kähler manifolds
}

\author{
Chandrashekar Devchand ${ }^{1}$ (D) Massimiliano Pontecorvo ${ }^{2} \cdot$ Andrea Spiro $^{3}$
}

Received: 17 February 2020 / Accepted: 29 June 2020 / Published online: 26 July 2020

(c) The Author(s) 2020

\begin{abstract}
We present a novel approach to the study of Yang-Mills instantons on quaternionic Kähler manifolds, based on an extension of the harmonic space method of constructing instantons on hyperkähler manifolds. Our results establish a bijection between local equivalence classes of instantons on quaternionic Kähler manifolds $M$ and equivalence classes of certain holomorphic maps on an appropriate $\mathrm{SL}_{2}(\mathbb{C})$-bundle over the Swann bundle of $M$.
\end{abstract}

Keywords Yang-Mills theory · Instantons · Quaternionic Kähler geometry · Harmonic space $\cdot$ Swann bundle $\cdot$ Hyperkähler cone

Mathematics Subject Classification $70 \mathrm{~S} 15 \cdot 53 \mathrm{C} 26 \cdot 14 \mathrm{~J} 60 \cdot 53 \mathrm{C} 28$

\section{Introduction}

Let $(M, g)$ be a $4 n$-dimensional quaternionic pseudo-Kähler (qk) manifold, by which we mean a pseudo-Riemannian manifold with holonomy in $\mathrm{Sp}_{1} \cdot \mathrm{Sp}_{p, q}, p+q=n$. Any such manifold is automatically Einstein and its constant scalar curvature is zero if and only if it is the quotient of a pseudo-hyperkähler (hk) manifold by some discrete group of isometries. In this paper, we are interested in studying instantons on $M$, that is to say in vector bundles equipped with connections, whose curvature tensor $F$ is pointwise invariant under the quaternionic structure of the tangent space. They are gauge fields which

Chandrashekar Devchand

devchand@aei.mpg.de

Massimiliano Pontecorvo

max@mat.uniroma3.it

Andrea Spiro

andrea.spiro@unicam.it

1 Max-Planck-Institut für Gravitationsphysik (Albert-Einstein-Institut), Am Mühlenberg 1, 14476 Potsdam, Germany

2 Dipartimento di Matematica e Fisica, Università Roma Tre, Largo San Leonardo Murialdo, 1, 00146 Rome, Italy

3 Scuola di Scienze e Tecnologie, Università di Camerino, Via Madonna delle Carceri, 62032 Camerino, Macerata, Italy 
minimise the Yang-Mills functional $Y M=\|F\|:=\int_{M} \operatorname{Tr} F \wedge * F$, simply because they saturate a topological bound on $Y M$, a property with a number of crucial consequences both in physics and mathematics. For an overview of the importance of instantons in mathematics, the reader might take a look at the beautiful lecture notes [31].

In a previous paper [13], we have considered instantons on hk manifolds and presented a differential geometric formulation of the harmonic space approach to them, a method that was originally developed in theoretical physics for studying supersymmetric field theories (see, e.g. [14, 16]). It is based on the notion of harmonic space $\mathcal{H}(N)$ for an hk manifold $(N, g)$, which is the trivial bundle $\mathcal{H}(N)=N \times \mathrm{SL}_{2}(\mathbb{C})$ over $N$ equipped with a non-product complex structure that can be described as follows. Each fibre $\{x\} \times \mathrm{SL}_{2}(\mathbb{C})$ of $\mathcal{H}(N)$ naturally projects onto the quotient $\{x\} \times \mathrm{SL}_{2}(\mathbb{C}) / B \simeq \mathbb{C} P^{1}$ by the Borel subgroup $B$ of $\mathrm{SL}_{2}(\mathbb{C})$ consisting of the upper triangular matrices. Thus, $\mathcal{H}(N)$ can be considered as a $B$-bundle over the twistor space $Z(N)=N \times \mathbb{C} P^{1}$ of the hk manifold $N$. The complex structure of $\mathcal{H}(N)$ is precisely the unique complex structure which makes $\mathcal{H}(N)$ a holomorphic bundle over $Z(N)$.

In [13], we provide full and complete proofs of the following correspondence, upon which the harmonic space method is founded. Starting from Yang-Mills data $(E, D)$ on an hk manifold $(N, h)$, where $E$ is a vector bundle associated with a principal $G$-bundle with connection $D$, we show there that $(E, D)$ is an instanton if and only if the corresponding pull-back $\left(E^{\prime}, D^{\prime}\right)$ over $\mathcal{H}(N)$ admits a distinguished class of local trivialisations (= gauges) of $E^{\prime}$ called analytic gauges, defined by the vanishing of certain components of the gauge potential $A^{\prime}$ of the connection $D^{\prime}$. In these gauges, all the remaining components of $A^{\prime}$ can be uniquely reconstructed from just one of them, the so-called prepotential, a holomorphic function taking values in the complexification $\mathfrak{g}^{\mathbb{C}}$ of the Lie algebra of the structure group $G$. Further, any holomorphic map satisfying a certain simple first-order equation on an $\mathrm{SL}_{2}(\mathbb{C})$-invariant open domain $\mathcal{W} \subset \mathcal{H}(N)$ is a prepotential for a (locally defined) instanton on $N$. Combining these results establishes a bijective correspondence between a special class of normalised prepotentials on $\mathcal{W}$ and the moduli space of locally defined instantons on $N$.

In the present paper, we establish a similar bijection for instantons on strict qk manifolds, i.e. those having nonzero scalar curvature. The notion of the Swann bundle $\pi: \mathcal{S}(M) \rightarrow M$ of a (strict) qk manifold $(M, g)$ [29] is crucial for our discussion. This is an $\mathbb{1}^{*} / \mathbb{Z}_{2}$-bundle over $M$, canonically determined by the qk structure and naturally equipped with a distinguished pseudo-Riemannian metric $h$ of conical type and a triple $\left(J_{\alpha}\right)_{\alpha=1,2,3}$ of integrable complex structures. It is thus an hk manifold, the so-called hyperkähler cone of $M$ [25] (see also [5, 17, 18]). Following [13], we may define its harmonic space $\mathcal{H}(\mathcal{S}(M))$ as the trivial $\mathrm{SL}_{2}(\mathbb{C})$-bundle over $\mathcal{S}(M)$. This naturally fibres over $M$, with fibres $\mathrm{SL}_{2}(\mathbb{C}) \ltimes \mathbb{M}^{*} / \mathbb{Z}_{2}$. We call this principal bundle $\mathcal{H}(\mathcal{S}(M)) \rightarrow M$ the harmonic space of the qk manifold $(M, g)$. Now, a Yang-Mills field $(E, D)$ on $(M, g)$ may successively be lifted to the hk manifold $\left(\mathcal{S}(M), h, J_{\alpha}\right)$ and to its harmonic space $\mathcal{H}(\mathcal{S}(M))$. Then, applying our previous results on instantons on hk manifolds [13] and imposing an $\mathbb{U}^{*} / \mathbb{Z}_{2}$-symmetry, we obtain the desired bijection between equivalence classes of certain holomorphic maps and equivalence classes of locally defined instantons on a strict qk manifold $M$.

This paper is structured as follows. After some preliminaries (Sect. 2), we provide (in Sect. 3) our presentation of the Swann bundle and discuss some of its properties which we shall need. In Sects. 4 and 5, we prove the above-described results on the correspondence between prepotentials and equivalence classes of instantons. In the final 
section (Sect. 6), we use the classical results of Narasimhan and Ramanan [21] on gauge fields with compact structure groups to express our solutions in a form reminiscent of the ADHM construction, showing that any local equivalence class of instantons is uniquely associated with a corresponding finite set of (locally defined) smooth matrixvalued maps $\lambda: \mathcal{U} \subset M \rightarrow \operatorname{Mat}_{k \times m}(\mathbb{C})$. This yields a more compact representation of the instanton prepotentials, which we expect will facilitate the further investigation of the moduli space of instantons on compact qk manifolds. We conclude with a discussion in our framework of the explicit examples of instantons on $\mathbb{H} P^{n}$ considered by Mamone Capria and Salamon [20].

\section{Preliminaries on quaternionic Kähler manifolds and instantons}

In this section, in order to fix our notation and definitions, we review the fundamental properties of quaternionic Kähler manifolds and of gauge fields, which we shall need in this paper.

\subsection{Quaternionic Kähler manifolds}

Let $W$ be a $4 n$-dimensional real vector space. A hypercomplex structure on $W$ is a triple $\left(I_{1}, I_{2}, I_{3}\right)$ of endomorphisms satisfying the multiplicative relations of the imaginary quaternions, $I_{\alpha}^{2}=-\operatorname{Id}_{W}$ and $I_{\alpha} I_{\beta}=I_{\gamma}$ for all cyclic permutations $(\alpha, \beta, \gamma)$ of $(1,2,3)$. This notion has the following counterpart in the category of manifolds: a hypercomplex structure on a $4 n$-dimensional real manifold $M$ is a triple $\left(J_{1}, J_{2}, J_{3}\right)$ of integrable complex structures on $M$, with the property that each triple $\left(I_{\alpha}:=\left.J_{\alpha}\right|_{x}\right), x \in M$, is a hypercomplex structure on $T_{x} M$.

These definitions admit the following generalisations. A quaternionic structure on $W$ is a 3-dimensional space of endomorphisms $Q_{W}=\operatorname{span}_{\mathbb{R}}\left(I_{1}, I_{2}, I_{3}\right) \subset$ End $(W)$ spanned by the three elements $I_{\alpha}$ of a hypercomplex structure. A quaternionic pseudo-Kähler structure on $W$ is an inner product $\langle\cdot, \cdot\rangle$ on $W$ which is Hermitian with respect to $Q_{W}$, meaning that every $J \in Q_{W}$ is skew-symmetric with respect to $\langle\cdot, \cdot\rangle$. The corresponding definition for manifolds is as follows.

Definition 2.1 A $4 n$-dimensional pseudo-Riemannian manifold $(M, g)$ of signature $(4 p, 4 q), p+q=n$, is a quaternionic pseudo-Kähler ( $q k)$ manifold if it admits a subbundle $Q \subset$ End $(T M)$ of quaternionic structures on the tangent spaces satisfying the following two conditions:

(i) each inner product $g_{x}$ is Hermitian with respect to the corresponding quaternionic structure $Q_{x}$;

(ii) the parallel transport of the Levi-Civita connection $\nabla$ of $g$ preserves $Q$.

Further, $(M, g)$ is a pseudo-hyperkähler $(h k)$ manifold if there exist three global $\nabla$-parallel sections $J_{1}, J_{2}, J_{3}$ of $Q$ (which are thus integrable complex structures) giving a hypercomplex structure on each tangent space of $M$.

It is well known that a qk manifold is Einstein and that its scalar curvature is zero if and only if it is locally isometric to an hk manifold. A qk manifold that is not locally hk, i.e. not Ricci flat, is called a strict qk manifold. 
Let $(M, g, Q)$ be a $4 n$-dimensional qk manifold. For each $x \in M$, an adapted frame of $T_{x} M$ is an orthonormal frame $\left(e_{i}\right) \subset T_{x} M$, for which there is a hypercomplex structure $\left(J_{1}, J_{2}, J_{3}\right) \subset Q_{x}$ such that

$$
e_{(4 k+1)+\alpha}=J_{\alpha} e_{4 k+1}, \quad \text { for } k=0, \ldots, n-1 \text { and } \alpha=1,2,3 .
$$

The adapted frames form a principal bundle $\pi: O_{g}(M, Q) \rightarrow M$ with structure group $\mathrm{Sp}_{1} \cdot \mathrm{Sp}_{p, q}$. It is preserved by the Levi-Civita connection and is a holonomic reduction of the orthonormal frame bundle $\pi^{\prime}: O_{g}(M) \rightarrow M$ of $(M, g)$.

Each adapted frame yields a convenient identification of the complexified tangent space $T_{x}^{\mathbb{C}} M$ with a tensor product of the form $\mathbf{H}_{x} \otimes \mathbf{E}_{x}$, with $\mathbf{H}_{x} \simeq \mathbb{C}^{2}$ and $\mathbf{E}_{x} \simeq \mathbb{C}^{2 n}$, first considered by Salamon [26]. To see this consider first, as the standard representation of $\mathrm{Sp}_{1} \cdot \mathrm{Sp}_{p, q}$ on $\mathbb{H}^{n}=\mathbb{C}^{2 n}$, the one for which the element $\left(\begin{array}{cc}i & 0 \\ 0 & -i\end{array}\right) \in \mathfrak{s p}_{1}$ acts on $\left(\mathbb{W}^{n}\right)^{\mathbb{C}} \simeq \mathbb{C}^{2} \otimes \mathbb{C}^{2 n}$ as the left multiplication by the imaginary unit $\mathbf{i} \in \mathbb{H}$. Now, if we use an adapted frame $v=\left.\left(e_{i}\right) \in O_{g}\left(M, J_{\alpha}\right)\right|_{x}$ to make the identification $T_{x} M \simeq \mathbb{R}^{4 n}=\mathbb{H}^{n}$, the standard left action of $\mathrm{Sp}_{1} \cdot \mathrm{Sp}_{p, q}$ on $\mathbb{\boxplus}^{n}$ corresponds to a left action of $\mathrm{Sp}_{1} \cdot \mathrm{Sp}_{p, q}$ on $T_{x} M$. By the above assumption, this action is such that $\left(\begin{array}{cc}i & 0 \\ 0 & -i\end{array}\right)$ acts on $T_{x} M$ precisely as the action of complex structure $I=v_{*}(\mathbf{i})$. This $\mathrm{Sp}_{1} \cdot \mathrm{Sp}_{p, q}$-action extends by $\mathbb{C}$-linearity to the standard action of $\mathrm{SL}_{2}(\mathbb{C}) \cdot \mathrm{Sp}_{n}(\mathbb{C})$ on $\left(\mathbb{T}^{n}\right)^{\mathbb{C}} \simeq \mathbb{C}^{2} \otimes \mathbb{C}^{2 n}$. Consequently, the $\mathbb{C}$-linear extension $v:\left(\mathbb{T}^{n}\right)^{\mathbb{C}} \rightarrow T_{x}^{\mathbb{C}} M$ of the considered adapted frame yields an $\mathrm{SL}_{2}(\mathbb{C}) \cdot \mathrm{Sp}_{n}(\mathbb{C})$ -equivariant isomorphism $T_{x}^{\mathbb{C}} M \simeq \mathbf{H}_{x} \otimes \mathbf{E}_{x}$ with $\mathbf{H}_{x} \simeq \mathbb{C}^{2}$ and $\mathbf{E}_{x} \simeq \mathbb{C}^{2 n}$. Any two such isomorphisms are necessarily related to each other by the action of an element of $\mathrm{Sp}_{1} \cdot \mathrm{Sp}_{p, q} \subset \mathrm{SL}_{2}(\mathbb{C}) \cdot \mathrm{Sp}_{n}(\mathbb{C})$, which clearly preserves the tensor product structure $\mathbf{H}_{x} \otimes \mathbf{E}_{x}$. Thus, modulo the action of this group, the identification of $T_{x}^{\mathbb{C}} M$ with $\mathbf{H}_{x} \otimes \mathbf{E}_{x}$ is independent of the chosen adapted frame $v=\left(e_{i}\right)$.

\subsection{Gauge fields and potentials}

Let $p: P \rightarrow M$ be a principal $G$-bundle and $p^{E}: E=P \times_{G, \rho} V \rightarrow M$ an associated vector bundle with fibre $V \simeq \mathbb{R}^{N}$, determined by a faithful linear representation $\rho: G \rightarrow \operatorname{GL}(V)$

Following physics terminology, a gauge is a local trivialisation $\varphi:\left.P\right|_{\mathcal{U}} \rightarrow \mathcal{U} \times G$ of $P$ over an open set $\mathcal{U}$. We call $\mathcal{U}$ the domain of the gauge. Given two gauges $\varphi, \varphi^{\prime}$ on overlapping domains $\mathcal{U}, \mathcal{U}^{\prime}$, the associated transition function is a map of the form $\left(\varphi^{\prime} \circ \varphi^{-1}\right)(x, h)=\left(x, g_{x} \cdot h\right)$ for a smooth map $g: \mathcal{U} \cap \mathcal{U}^{\prime} \rightarrow G$. The gauge transformation corresponding to this transition function is the smooth family of $G$-automorphisms $h \mapsto g_{x} \cdot h, x \in \mathcal{U} \cap \mathcal{U}^{\prime}$.

Given a connection 1-form $\omega$ on $P$, there is a uniquely associated covariant derivative $\underset{\sim}{D}$ for the sections of $E=P \times_{G, \rho} V$, locally defined as follows. Consider a gauge $\left.P\right|_{\mathcal{U}} \stackrel{\sim}{\rightarrow} \mathcal{U} \times G$ and use it to identify each tangent space $T_{x} P, x \in \mathcal{U}$, with a direct sum $T_{(x, g)}(\mathcal{U} \times G) \simeq T_{x} \mathcal{U}+T_{g} G$. Then, the connection 1-form $\omega$ is pointwise identifiable with a sum of the form $\left.\omega\right|_{(x, g)}=-A_{(x, g)}+\omega_{g}^{G}$, where $\omega^{G}$ is the Maurer-Cartan form of $G$ and each map $A_{(\cdot, g)}: \mathcal{U} \rightarrow T^{*} \mathcal{U} \otimes \mathfrak{g}$ is a $\mathfrak{g}$-valued 1-form, changing $G$-equivariantly with respect to $g$. The 1 -form $A:=A_{(\cdot, e)}: \mathcal{U} \rightarrow T^{*} M \otimes \mathfrak{g}$ is called the potential of $\omega$ in the considered gauge. If $\left.P\right|_{\mathcal{U}} \simeq \mathcal{U}^{\prime} \times G$ is a different gauge on a domain $\mathcal{U}^{\prime}$ that overlaps with $\mathcal{U}$ and 
$h \mapsto g_{x} \cdot h$ is the corresponding gauge transformation, then the potential of $\omega$ in this new gauge is given by

$$
A^{\prime}=\operatorname{Ad}_{g^{-1}} A+g^{-1} d g \quad \text { on } \mathcal{U} \cap \mathcal{U}^{\prime} .
$$

Now, if $\sigma:\left.\mathcal{U} \rightarrow E\right|_{\mathcal{U}} \simeq \mathcal{U} \times V$ is a (local) section of $E=P \times_{G, \rho} V$ of the form $\sigma(x)=\left(x, s^{i}(x)\right)$ for a smooth map $\left(s^{i}\right): \mathcal{U} \rightarrow V=\mathbb{R}^{N}$, then the covariant derivative of $\sigma$ along a vector field $X \in \mathfrak{X}(\mathcal{U})$ is the section $D_{X} \sigma$ of $\left.E\right|_{\mathcal{U}} \simeq \mathcal{U} \times V$ defined by

$$
D_{X} \sigma(x)=\left(x,\left.\left(X \cdot \sigma^{i}\right)\right|_{x}+\left.A_{j}^{i}(X) \sigma^{j}\right|_{x}\right),
$$

where $A_{j}^{i}:=\rho_{*} \circ A$, with $\rho_{*}: \mathfrak{g} \rightarrow \mathfrak{g l}(V)$ being the Lie algebra representation determined by the linear representation $\rho: G \rightarrow \mathrm{GL}(V)$.

Given a Lie group $G$, we shall call a pair $(E, D)$ consisting of

(1) a vector bundle $E$, associated with a principal $G$-bundle $p: P \rightarrow M$ and

(2) a covariant derivative $D$ on $E$, induced by a connection $\omega$ on $P$

a gauge field with structure group $G$ and we shall say that $(E, D)$ is associated with $(P, \omega)$. Consider a complex Lie group $G$ with compact real form $G^{o} \subset G$. If $p: P \rightarrow M$ and $\omega$ admit reductions to a $G^{o}$-subbundle $P^{o} \subset P$ and a connection $\omega^{o}$ on $P^{o}$, we say that the pair $(P, \omega)$ is reducible to $\left(P^{o}, \omega^{o}\right)$ and that the gauge field $(E, D)$ is the complexification of a gauge field with compact structure group $G^{o}$.

In a gauge $\varphi:\left.P\right|_{\mathcal{U}} \rightarrow \mathcal{U} \times G$ and its associated gauge $\hat{\varphi}$ for $E=P \times_{G, \rho} V$,

$$
\left.\hat{\varphi}:\left.E\right|_{\mathcal{U}} \rightarrow \mathcal{U} \times V, \quad \hat{\varphi}\left(\left[\varphi^{-1}(x, e), v\right)\right]\right):=(x, v),
$$

the curvature tensors of $\omega$ and $D$ are, respectively, the ( $\mathfrak{g}$ - and $\mathfrak{g l}(V)$-valued) 2-forms $\mathcal{F}^{\varphi}$ and $F_{x}$ on $\mathcal{U}$, defined by

$$
\mathcal{F}_{x}^{\varphi}(v, w):=2 \Omega_{(x, e)}(v, w), \quad F_{x}(v, w):=\rho_{*} \circ \mathcal{F}_{x}^{\varphi}(v, w), \quad \text { for } v, w \in T_{x} \mathcal{U},
$$

where $\Omega$ is the curvature 2 -form of the connection 1 -form $\omega$. Note that $\mathcal{F}^{\varphi}$ is expressed in terms of the potential $A$ by

$$
\mathcal{F}^{\varphi}(X, Y)=X \cdot(A(Y))-Y \cdot(A(X))+[A(X), A(Y)]-A([X, Y]) .
$$

The curvature $F$ of $D$ is independent of the considered gauge and it satisfies the identity

$$
F(X, Y) s=\left[D_{X}, D_{Y}\right] s-D_{[X, Y]} s \quad \text { for sections } s \in \Gamma(E),
$$

a property that is often used as an alternative definition for $F$.

The above notions have analogues in the category of holomorphic bundles. If $(M, J)$ is a complex manifold, $G$ a complex Lie group and $V$ a complex vector space, then a principal $G$-bundle $p: P \rightarrow M$ (resp. a complex vector bundle $p: E \rightarrow M$ with fibre $V$ ) is called holomorphic if it is equipped with a complex structure $J$, such that the right action of $G$ on $P$ (resp. the vector bundle structure on $E$ ) is $\widehat{J}$-holomorphic and the projection $p$ is a $(\widehat{J}, J)$ -holomorphic mapping. A gauge is called holomorphic if it is a local holomorphic map from $(P, \widehat{J})($ resp. $(E, \widehat{J}))$ to the cartesian product $M \times G$ (resp. $M \times V)$, equipped with the product complex structure. 
A connection form $\omega$ on a holomorphic $G$-bundle $(P, \widehat{J})$ is called $\widehat{J}$-invariant if the corresponding horizontal spaces in $T P$ are invariant under the complex structure $\widehat{J}$. This is the case if and only if in any holomorphic gauge $\varphi:\left.P\right|_{\mathcal{U}} \rightarrow \mathcal{U} \times G$, the potential $A: \mathcal{U} \rightarrow T^{*} \mathcal{U} \otimes \mathfrak{g}$ takes values in $T^{10 *} M \otimes \mathfrak{g}^{10}+T^{01 *} M \otimes \mathfrak{g}^{01}$, where, $T^{10} M, T^{01} M$ are the holomorphic and anti-holomorphic distributions of $M$ and $\mathfrak{g}^{10}, \mathfrak{g}^{01}$ are the subalgebras of $\mathfrak{g}^{\mathbb{C}}$ (both isomorphic to $\mathfrak{g}$ ), which are generated by the vectors of type $(1,0)$ and $(0,1)$. The $\mathfrak{g}$-valued 1 -forms $A_{x}, x \in \mathcal{U}$, being real, the potential $A$ is uniquely determined by the $(1,0)$-potential $A^{10}: \mathcal{U} \rightarrow T^{10 *} M \otimes \mathfrak{g}^{10}$.

\subsection{Instantons on quaternionic Kähler manifolds}

Let $(M, g, Q)$ be a qk manifold and consider the isomorphisms $T_{x}^{\mathbb{C}} M \simeq \mathbf{H}_{x} \otimes \mathbf{E}_{x}$ described in Sect. 2.1. The space of complex 2-forms $\Lambda^{2} T_{x}^{* \mathbb{C}} M$ splits into three irreducible $\mathrm{SL}_{2}(\mathbb{C}) \cdot \mathrm{Sp}_{n}(\mathbb{C})$ modules:

$$
\Lambda^{2} T_{x}^{* \mathbb{C}} M \simeq\left(\mathbb{C} \omega_{\mathbf{H}_{x}}\right) \otimes S^{2} \mathbf{E}_{x}^{*}+S^{2} \mathbf{H}_{x}^{*} \otimes\left(\mathbb{C} \omega_{\mathbf{E}_{x}}\right)+S^{2} \mathbf{H}_{x}^{*} \otimes \Lambda_{0}^{2} \mathbf{E}_{x}^{*} .
$$

Here, $\omega_{\mathbf{H}_{x}}$ and $\omega_{\mathbf{E}_{x}}$ are the $\mathrm{SL}_{2}(\mathbb{C})$ and $\mathrm{Sp}_{n}(\mathbb{C})$ invariant symplectic forms of $\mathbf{H}_{x}$ and $\mathbf{E}_{x}$, respectively, and $\Lambda_{0}^{2} \mathbf{E}_{x}$ is the irreducible $\operatorname{Sp}_{n}(\mathbb{C})$-submodule of $\Lambda^{2} \mathbf{E}_{x}$ that is complementary to $\mathbb{C} \omega_{\mathbf{E}_{r}}$. The decomposition (2.7) is independent of the considered isomorphism $T_{x}^{\mathbb{C}} M \simeq \mathbf{H}_{x} \otimes \mathbf{E}_{x}$, since the latter is unique up to the action of an element in $\mathrm{Sp}_{1} \cdot \mathrm{Sp}_{p, q}$.

The (C-linear extension of the) curvature tensor $F_{x}$ of a gauge field $(E, D)$ decomposes according to (2.7). We write

$$
\begin{aligned}
F_{x}=F_{x}^{(1)}+F_{x}^{(2)} \text { with } \quad F_{x}^{(1)} & \in \mathbb{C} \omega_{\mathbf{H}_{x}} \otimes S^{2} \mathbf{E}_{x}^{*} \otimes \operatorname{End}\left(E_{x}\right) \\
F_{x}^{(2)} & \in\left(S^{2} \mathbf{H}_{x}^{*} \otimes\left(\mathbb{C} \omega_{\mathbf{E}_{x}}+\Lambda_{0}^{2} \mathbf{E}_{x}^{*}\right)\right) \otimes \operatorname{End}\left(E_{x}\right) .
\end{aligned}
$$

A gauge field $(E, D)$ on a qk manifold is called an instanton if the $F^{(2)}$ component of its curvature tensor vanishes identically. Such instantons provide minima of the Yang-Mills functional $\int_{M}|F|^{2} \omega_{g}$ and are thus Yang-Mills fields. Instanton equations on quaternionic Kähler manifolds have been studied by several authors, e.g. [7, 20, 23, 30, 32], and are examples of a larger class of algebraic equations for the curvature tensor which imply the Yang-Mills equations [1, 6, 8].

If an instanton $(E, D)$ has a compact (resp. complex) structure group, we call it an instanton of compact (resp. complex) type.

\section{The Swann bundle of a quaternionic Kähler manifold}

\subsection{Conformally adapted frames}

Let $(M, g, Q)$ be a $4 n$-dimensional qk manifold. Note that each homothetic Riemannian manifold $(M, r g)$ determined by a homothetic parameter $r \in(0,+\infty)$ is also a qk manifold provided it is associated with the same bundle of quaternionic structures $Q \subset$ End $(T M)$ as $(M, g, Q)$. We define the bundle of conformally adapted frames of $(M, g)$, denoted $\mathrm{CO}_{g}(M, Q)$, as the union of all adapted frame bundles of all its associated homothetic manifolds, 


$$
p: \mathcal{C} O_{g}(M, Q):=\bigcup_{r \in(0,+\infty)} O_{r g}(M, Q) \longrightarrow M .
$$

This is a principal bundle with structure group

$$
\mathbb{R}_{>0} \times \mathrm{Sp}_{1} \cdot \mathrm{Sp}_{p, q}=\mathbb{R}^{*} \times_{\mathbb{Z}_{2}}\left(\mathrm{Sp}_{1} \times_{\mathbb{Z}_{2}} \mathrm{Sp}_{p, q}\right) .
$$

For each homothetic metric $r g$, the canonical embedding of the bundle of adapted frames into the bundle of orthonormal frames $O_{r g}(M), t_{r}: O_{r g}(M, Q) \hookrightarrow O_{r g}(M)$, can be used to pull-back the tautological 1-form $\Theta^{(r)}$ and the Levi-Civita connection form $\Omega^{(r) L C}$ of $O_{r g}(M)$ onto $O_{r g}(M, Q)$. We thus have two canonically defined 1-forms,

$$
\vartheta^{(r)}:=\iota_{r}^{*} \Theta^{(r)} \text { and } \omega^{(r)}:=\imath_{r}^{*} \Omega^{(r) L C},
$$

the first being $\mathbb{R}^{n}$-valued and the second $\mathfrak{S} \mathfrak{p}_{1}+\mathfrak{S} \mathfrak{p}_{n}$-valued. Although these 1-forms clearly depend on the homothetic parameter $r$, they combine nonetheless to determine two smooth 1 -forms $\vartheta$ and $\omega$ on $\mathcal{C} O_{g}(M, Q)$. We call them the tautological 1-form and the Levi-Civita connection 1-form of $\mathcal{C O}_{g}(M, Q)$.

Note that:

- $\vartheta$ is the natural tautological 1-form of $\mathcal{C O}_{g}(M, Q)$ provided that this bundle is considered to be a subbundle of the bundle of linear frames $L(M)$ of $M$;

- $\omega$ is a connection 1-form on $\mathcal{C} O_{g}(M, Q)$ provided that $\mathfrak{S \mathfrak { p } _ { 1 }}+\mathfrak{S p}_{n}$ is considered to be a

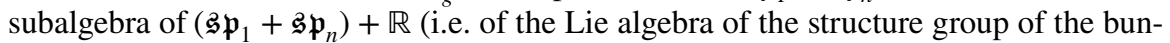
dle of conformally adapted frames).

\subsection{The Swann bundle and its canonical structure of a hyperkähler cone}

The Swann bundle of a qk manifold $(M, g, Q)$ is the principal bundle

$$
\pi: \mathcal{S}:=\mathcal{C} O_{g}(M, Q) / \operatorname{Sp}_{p, q} \rightarrow M
$$

with structure group $\mathbb{H}^{*} / \mathbb{Z}_{2}\left(\simeq \mathbb{R}^{*} \times_{\mathbb{Z}_{2}}\left(\mathrm{Sp}_{1} \times_{\mathbb{Z}_{2}} \mathrm{Sp}_{p, q}\right) / \mathrm{Sp}_{p, q}\right)$.

As we will shortly see, this bundle admits a very special hypercomplex structure $\left(J_{1}, J_{2}\right.$, $\left.J_{3}\right)$ and a distinguished pseudo-Riemannian metric $h$ which make the tuple $\left(\mathcal{S}, h, J_{1}, J_{2}, J_{3}\right)$ an hk manifold canonically associated with $(M, g, Q)$. This hk manifold plays a crucial role in our discussion. The explicit construction of the quadruple of tensor fields $\left(h, J_{1}, J_{2}, J_{3}\right)$ is as follows.

For each point $(x,[u]) \in \mathcal{S}=\left.\mathcal{C} O_{g}(M, Q)\right|_{x} / \operatorname{Sp}_{p, q}, x \in M$, consider the $\Vdash$-valued 1 -form

$$
\omega_{(x,[u])}^{\mathcal{S}}:=\left(\sigma^{*}(\omega)_{(x,[u])}\right)^{\mathbb{A}}: T_{(x, q)} \mathcal{S} \longrightarrow \mathbb{H}, \quad \text { where }
$$

(a) $\sigma: \mathcal{U} \subset \mathcal{S} \rightarrow \mathcal{C} O_{g}(M, Q)$ is a local section, defined on a neighbourhood of $(x,[u])$, of the $\operatorname{Sp}_{p, q}$-bundle $\mathcal{C} O_{g}(M, Q) \rightarrow \mathcal{S}$ over the Swann bundle $\mathcal{S}$,

(b) $(\cdot)^{\mathbb{A}}: \mathbb{R}+\mathfrak{S} \mathfrak{p}_{1}+\mathfrak{S} \mathfrak{p}_{p, q} \rightarrow \mathbb{U}$ denotes the canonical projection of $\mathbb{R}+\mathfrak{S} \mathfrak{p}_{1}+\mathfrak{S} \mathfrak{p}_{p, q}$ onto $\mathbb{R}+\mathfrak{S p}_{1} \simeq \mathbb{W}$. 
Note that since the subalgebra $\mathbb{H}=\mathbb{R}+\mathfrak{S} \mathfrak{p}_{1}$ commutes with the subalgebra $\mathfrak{s \mathfrak { p } _ { p , q }}$ of the Lie algebra of the structure group of $p: \mathcal{C} O_{g}(M, Q) \rightarrow \mathcal{S}$, the component $\omega^{\mathbb{E}}$ of the connection form $\omega$ is $\mathrm{Sp}_{p, q}$-invariant. This implies that the mapping (3.1) is well defined and independent of the choice of $\sigma$. Further, the collection of 1 -forms $\omega_{(x,[u])}^{\mathcal{S}}$ determines a smooth $\Vdash-$-valued 1 -form $\omega^{\mathcal{S}}$, which is in fact a connection form on the $\mathbb{U}^{*} / \mathbb{Z}_{2}$-bundle $\mathcal{S}$.

The $\mathbb{U}^{*} / \mathbb{Z}_{2}$-invariant horizontal distribution $\mathcal{D} \subset T \mathcal{S}$, given by the kernel of the 1 -form $\omega^{\mathcal{S}}$, is naturally equipped with a quadruple of tensor fields $g^{\mathcal{D}}, J_{\alpha}^{\mathcal{D}}, \alpha=1,2,3$, defined pointwise as follows. For each $\left.(x,[u]) \in \mathcal{S}\right|_{x}, x \in M$, we introduce:

- the tensor field in $\mathcal{D}_{(x,[u])}^{*} \otimes \mathcal{D}_{(x,[u])}^{*}$ given by

$$
g_{(x,[u])}^{\mathcal{D}}(v, w):=r g_{x}\left(\pi_{*}(v), \pi_{*}(w)\right)
$$

- the tensor field in $\mathcal{D}_{(x,[u])} \otimes \mathcal{D}_{(x,[u])}^{*}$ given by

$$
\left.J_{\alpha}^{\mathcal{D}}\right|_{(x,[u])}(v):=\left(\left.\pi_{*}\right|_{\mathcal{D}}\right)^{-1}\left(J_{\alpha}^{(u)}\right),
$$

where $\left(J_{\alpha}^{(u)}\right)$ is the unique triple of complex structures of $T_{x} M$ satisfying (2.1) for a frame $u=\left(e_{i}\right)$ in equivalence class $[u]$. It is possible to check that such a triple does not depend on the particular adapted frame $u=\left(e_{i}\right)$ chosen out of the equivalence class $[u]$, meaning that (3.3) is well defined. Using the above construction, we may verify the following (see, e.g. [17, 25] for details):

(a) the action of the subgroup $\left\{e^{t}, t \in \mathbb{R}\right\}$ of $\mathbb{H}^{*} / \mathbb{Z}$ leaves invariant each of the tensor fields $J_{\alpha}$, but not the tensor field $g^{\mathcal{D}}$, which transforms as

$$
R_{e^{t}}^{*}\left(g^{\mathcal{D}}\right)=e^{t} g^{\mathcal{D}}
$$

(b) the right action $R_{[q]}: \mathcal{S} \rightarrow \mathcal{S}$ of each element of the structure group $\mathbb{U}^{*} / \mathbb{Z}_{2}$ of the form

$$
[q]=q \bmod \mathbb{Z}_{2} \in \mathrm{Sp}_{1} / \mathbb{Z}_{2} \subset \mathbb{M}^{*} / \mathbb{Z}_{2}
$$

leaves invariant $g^{\mathcal{D}}$, but not the triple $\left(J_{1}^{\mathcal{D}}, J_{2}^{\mathcal{D}}, J_{3}^{\mathcal{D}}\right)$, which get rotated in the following sense: at each point $\left.(x,[u]) \in \mathcal{S}\right|_{x}$, the triple $\left(R_{[q] *}\left(\left.J_{\alpha}^{\mathcal{D}}\right|_{(x,[u])}\right)\right)_{\alpha=1,2,3}$ is the triple of complex structures on $\mathcal{D}_{(x,[u] \cdot[q])}$ projecting onto the triple of complex structures of $T_{x} M$

$$
\left.J_{\alpha}^{(q)}\right|_{x}:=\left.R^{\left(q^{-1}\right)} \circ J_{\alpha}\right|_{x} \circ R^{(q)},
$$

where $R^{(q)}$ is the right $\mathrm{Sp}_{1}$-action

$$
R^{(q)}: T_{x} M \longrightarrow T_{x} M, \quad R^{(q)}(v):=v \cdot q,
$$

determined by one of the isomorphisms $T_{x} M \simeq \mathbb{T}^{n}$ established by an adapted frame $u=\left(e_{i}\right)$ in the equivalence class $[u]$. It can be directly checked that the complex structures (3.5) are independent of the choices of $q \in[q]$ and $u \in[u]$.

We now canonically "extend" the tensor fields $g^{\mathcal{D}}$ and $J_{\alpha}^{\mathcal{D}}$, which we have so far defined only on vectors of the distribution $\mathcal{D} \subset T \mathcal{S}$, to all of $T \mathcal{S}$. First recall that each 1-form $\left.\omega^{\mathcal{S}}\right|_{(x,[u])}$ gives an isomorphism between the vertical tangent space $\mathcal{V}_{(x,[u])}=T_{(x,[u])}^{V} \mathcal{S}$ and $\mathbb{U}=\operatorname{Lie}\left(\mathbb{M}^{*} / \mathbb{Z}_{2}\right)$. It therefore induces a natural hypercomplex structure $\left(\left.J_{\alpha}^{\mathcal{V}}\right|_{(x,[u])}\right)$ on $\mathcal{V}_{(x,[u])}$ corresponding to the 
standard hypercomplex structure of $\mathbb{H}$, i.e. $\left(\left.J_{1}^{\mathcal{V}}\right|_{(x,[u])}:=\mathbf{i},\left.J_{2}^{\mathcal{V}}\right|_{(x,[u])}:=\mathbf{j}, J_{3}^{\left.\mathcal{V}\right|_{(x,[u]}}:=\mathbf{k}\right)$. We thus have a triple $\left(\mathbb{J}_{\alpha}\right)_{\alpha=1,2,3}$ of almost complex structures on $\mathcal{S}$ defined at each point $(x,[u])$ by

$$
\left.\mathbb{J}_{\alpha}\right|_{(x,[u])}=\left.J_{\alpha}^{\mathcal{D}}\right|_{(x,[u])}+\left.J_{\alpha}^{\mathcal{V}}\right|_{(x,[u])} .
$$

The bundle $\mathcal{U}_{n /(n+1)}(M) \rightarrow M$, considered by Pedersen et al. [25] (or, equivalently, the bundle $\mathbf{H}^{\prime} \rightarrow M$ considered in[27]) is identifiable with the Swann bundle $\mathcal{S} \rightarrow M$ defined above and the triple (3.6) coincides with the triple of almost complex structures defined there. Thus, by Prop. 3.4 of [25], we immediately have the following

Proposition 3.1 ([25]) The almost complex structures (3.6) are integrable and form a hypercomplex structure on $\mathcal{S}$ satisfying the following conditions:

(1) The right action of the subgroup $\left\{e^{t}, t \in \mathbb{R}\right\} \subset \mathbb{U}^{*} / \mathbb{Z}_{2}$ leaves each $\mathbb{J}_{\alpha}$ invariant, whereas the right action of $\mathrm{Sp}_{1} / \mathbb{Z}_{2} \subset \mathbb{H}^{*} / \mathbb{Z}_{2}$ rotates them according to (3.5).

(2) The distributions $\mathcal{V}, \mathcal{D}$ are invariant under each complex structure $\mathbb{J}_{\alpha}$.

(3) Along every fibre $\left.\mathcal{S}\right|_{x} \simeq \mathbb{M}^{*} / \mathbb{Z}_{2}$, the triple $\left.\left(\mathbb{J}_{1}, \mathbb{J}_{2}, \mathbb{J}_{3}\right)\right|_{\mathcal{S}_{x}}$ is equal to $(\mathbf{i}, \mathbf{j}, \mathbf{k})$.

(4) For each $\left.(x,[u]) \in \mathcal{S}\right|_{x}$, the projection $\pi_{*}$ maps the triple $\left(\left.\mathbb{J}_{\alpha}\right|_{(x,[u])}\right)$ onto a triple $\left(J_{\alpha}\right)$ of complex structures of $T_{x} M$ belonging to the twistor bundle $\mathcal{Z}(M)$ of $(M, g, Q)$, thus establishing a $\mathbb{C}^{*}$-bundle $p: \mathcal{S} \rightarrow \mathcal{Z}(M)$ over $\mathcal{Z}(M)$.

Up to a bundle automorphism preserving $\omega^{\mathcal{S}}$, the triple $\left(\mathbb{J}_{1}, \mathbb{J}_{2}, \mathbb{J}_{3}\right)$ is the unique hypercomplex structure on $\mathcal{S}$ satisfying (1)-(4).

Having "extended" the triple of tensor fields $J_{\alpha}^{\mathcal{D}}$ in a canonical way, we now simi-

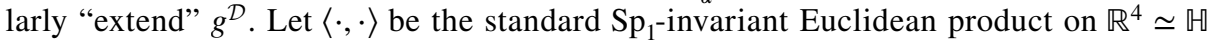
and on each vertical subspace $\mathcal{V}_{(x,[u])} \subset T_{(x,[u])} \mathcal{S}$, define the inner product

$$
g^{\mathcal{V}}(v, w)_{(x,[u])}:=\left\langle\omega^{\mathcal{S}}(v), \omega^{\mathcal{S}}(w)\right\rangle, \quad v, w \in T_{[u]}^{V} \mathcal{S} .
$$

If $(M, g, Q)$ is a strict qk manifold, i.e. with scalar curvature $\Lambda \neq 0$, the canonical Swann metric on $\mathcal{S}$ is the pseudo-Riemannian metric defined by

$$
h(X, Y)= \begin{cases}\frac{1}{\Lambda} g^{\mathcal{V}}(X, Y) & \text { if } X, Y \in \mathcal{V}, \\ 0 & \text { if } X \in \mathcal{V}, Y \in \mathcal{D}, \\ g^{\mathcal{D}}(X, Y) & \text { if } X, Y \in \mathcal{D} .\end{cases}
$$

If $(M, g, Q)$ is non-strict $(\Lambda=0)$, the canonical Swann metric on $\mathcal{S}$ is taken to be any metric having the form (3.7) with $\frac{1}{\Lambda}$ replaced by some positive constant $c>0$. For a canonical Swann metric $h$, the following hold:

(i) $h$ is Hermitian with respect to each of the three complex structures $\mathbb{J}_{\alpha}$.

(ii) If $(M, g)$ has signature $(4 p, 4 q)$, the signature of $h$ is $(4 p+4,4 q)$ if $\Lambda \geq 0$ and $(4 p, 4 q+4)$ if $\Lambda<0$.

(iii) $h$ is invariant under the right action of every $q \in \mathrm{Sp}_{1} / \mathbb{Z}_{2} \subset \mathbb{U}^{*} / \mathbb{Z}_{2}$.

(iv) For each $e^{t} \in \mathbb{Q}^{*} / \mathbb{Z}_{2}$, we have that $R_{e^{t}}^{*} h=e^{t} h$ (i.e. $h$ behaves as a conical metric with respect to the dilations $\left.e^{t}\right)$.

(v) The quadruple $\left(h, \mathbb{J}_{1}, \mathbb{J}_{2}, \mathbb{J}_{3}\right)$ is an hk structure on $\mathcal{S}$. 
Note that if $\Lambda=0$, property (v) is essentially a trivial consequence of the fact that $\mathcal{S}$ is a locally trivial bundle over $M$. If $\Lambda \neq 0,(\mathrm{v})$ is a non-trivial property, following from results in $[29, \S 3]$.

The hk manifold $\left(\mathcal{S}, h, \mathbb{J}_{1}, \mathbb{J}_{2}, \mathbb{J}_{3}\right)$ is called the hyperkähler $(h k)$ cone of the qk manifold $(M, g, Q)$.

\section{3 $\mathbb{U}^{*}$-conical manifolds and their relation to qk manifolds}

We have seen in the previous section that any (strict) qk manifold has a canonically associated hk manifold, namely its hk cone $\left(\mathcal{S}, h, \mathbb{J}_{\alpha}\right)$. This correspondence has an inverse. Let us first consider the following:

Definition 3.2 Let $\left(N, h, \mathbb{J}_{\alpha}\right)$ be an hk manifold. We say that it is $\mathbb{U}^{*}$-conical if it is equipped with an almost effective right action $R: \mathbb{H}^{*} \rightarrow \operatorname{Diff}(N)$, with $\operatorname{ker} R=\mathbb{Z}_{2}$, and displays the following properties.

(a) The effective right $\mathbb{U}^{*} / \mathbb{Z}_{2}$-action makes $N$ an $\mathbb{U}^{*} / \mathbb{Z}_{2}$-bundle over $M=N / \mathbb{U}^{*}$ such that the restrictions of $h$ and $\rrbracket_{\alpha}$ to the vertical tangent spaces render each fibre $N_{x}$, being 3-holomorphically isometric to $\left.\left(\mathbb{U}^{*} / \mathbb{Z}_{2}, c<\cdot, \cdot\right\rangle\right)$ for some constant $c$, independent of $x$.

(b) The distribution $\mathcal{D}$, given by the spaces that are $h$-orthogonal to the $\mathbb{H}^{*}$-orbits in $N$, is the horizontal distribution of a connection form on the bundle $N \rightarrow M=N / \mathbb{U}^{*}$.

(c) For each $\left\{e^{t}, t \in \mathbb{R}\right\} \subset \mathbb{U}^{*}$, the map $R_{e^{t}}: N \rightarrow N$ is $\mathbb{J}_{\alpha}$-holomorphic for each $\mathbb{J}_{\alpha}$, but it acts on the metric $h$ as a homothety, $R_{e^{t} *} h=e^{t} h$.

(d) For each $[q] \in \mathbb{U}^{*} / \mathbb{Z}_{2}$ of the form $[q]=q \bmod \mathbb{Z}_{2}$ for $q \in \mathrm{Sp}_{1} / \mathbb{Z}_{2}$, the map $R_{q}: N \rightarrow N$ acts on $h$ and $\mathbb{J}_{\alpha}$ as follows:

- it rotates the hypercomplex structure $\left(\mathbb{J}_{1}, \mathbb{J}_{2}, \mathbb{J}_{3}\right)$ as in (3.5)

- it leaves $\left.h\right|_{\mathcal{D} \times \mathcal{D}}$ invariant.

(e) If $H_{D}$ is the vector field on $N$ generating the 1-parameter family of diffeomorphisms $\Phi_{t}^{H_{D}}:=R_{e^{t}}$, the tensor field $\mathrm{c} \in \Lambda^{2} \mathcal{D}^{*}$ defined by

$\mathfrak{c}_{y}(v, w):=h_{y}\left(\nabla_{v} H_{D}, w\right)-h_{y}\left(\nabla_{w} H_{D}, v\right), \quad y \in N, \quad v, w \in \mathcal{D}_{y}$,

vanishes identically.

Note that (c) implies the invariance of the Levi-Civita connection $\nabla$ under diffeomorphisms $R_{e^{t}}$. Thus, condition (e) is equivalent to requiring that $\mathrm{c}$ vanishes identically on at least one hypersurface transversal to the orbits of $\left\{e^{i t}, t \in \mathbb{R}\right\}$.

The following theorem gives a characterisation of hk cones. It contains a reformulation of certain results in $[5,25,29]$.

Theorem 3.3 Let $\left(N, h, \mathbb{J}_{\alpha}\right)$ be a $4(n+1)$-dimensional $\mathbb{H}^{*}$-conical manifold and $\mathcal{V}^{\mathrm{Sp}_{1}}$ and $\mathcal{D}$ the distributions in TN consisting, respectively, of vectors tangent to the $\mathrm{Sp}_{1}$-orbits and those h-orthogonal to the $\mathbb{U}^{*}$-orbits. Then:

(i) The distribution $\mathcal{D}+\mathcal{V}^{\mathrm{Sp}_{1}}$ is involutive.

(ii) Given a maximal integral leaf $S \subset N$ of $\mathcal{V}^{\mathrm{Sp}_{1}}+\mathcal{D}$ and denoting $M:=S / \mathrm{Sp}_{1}$, define the pseudo-Riemannian metric $g$ and the bundle $Q \subset$ End (TM) over $M$ by 


$$
\begin{aligned}
& g_{x}(v, w):=h_{y}(v, w), \\
& Q_{x}:=\operatorname{span}_{\mathbb{R}}\left\{J=\left.\mathbb{J}_{\alpha}\right|_{\mathcal{D}_{y}}, y \in x=[y]\right\},
\end{aligned}
$$

for each $x=[y] \in M=S / \mathrm{Sp}_{1}$ (using of course the isomorphism $\pi_{*}: T S \rightarrow T M$ to identify each $\mathcal{D}_{y} \subset T_{y} S$ with $\left.T_{x=[y]} M\right)$. Then, $(M, g, Q)$ is a qk manifold, whose hk cone is 3-holomorphically isometric to $\left(N, h, \mathbb{J}_{\alpha}\right)$.

\subsection{A geometrical interpretation of the Swann bundle}

As discussed in Sect. 2.1, the adapted frames of a qk manifold $(M, g, Q)$ establish $\mathbb{C}$-linear isomorphisms between the complexified tangent spaces $T_{x}^{\mathbb{C}} M$ and $\mathbb{C}^{2} \otimes \mathbb{C}^{2 n}$ and make $T_{x}^{\mathbb{C}} M$ an $\left(\mathrm{SL}_{2}(\mathbb{C}) \times_{\mathbb{Z}_{2}} \mathrm{Sp}_{n}(\mathbb{C})\right)$-module of the form $T_{x}^{\mathbb{C}} M \simeq \mathbf{H}_{x} \otimes \mathbf{E}_{x}$, with

$$
\mathbf{H}_{x}=T_{x}^{\mathbb{C}} M / \mathrm{SL}_{2 n}(\mathbb{C}) \simeq \mathbb{C}^{2}, \quad \mathbf{E}_{x}=T_{x}^{\mathbb{C}} M / \mathrm{SL}_{2}(\mathbb{C}) \simeq \mathbb{C}^{2 n} .
$$

We recall that the isomorphism $T_{x}^{\mathbb{C}} M \simeq \mathbf{H}_{x} \otimes \mathbf{E}_{x}$ is independent of the adapted frame $u=\left(e_{i}\right)$ used in its construction. Thus, the family of vector spaces $\mathbf{H}_{x} \simeq \mathbb{C}^{2}, x \in M$, constitutes a rank two holomorphic vector bundle $\pi: \mathbf{H} \rightarrow M$, intrinsically determined by the quaternionic structure of $(M, g, Q)$ and locally equipped with a field $\omega_{\mathbf{H}}$ of $\mathrm{Sp}_{1}$-invariant symplectic 2-forms along the fibres (see [26]). The field $\omega_{\mathbf{H}}$ is uniquely determined up to multiplication by a nowhere vanishing function.

Now, let us denote by $\left(h_{1}^{o}, h_{2}^{o}\right),\left(e_{a}^{o}\right)$ and $\left(e_{i a}^{o}\right)$ the standard bases for $\mathbb{C}^{2}, \mathbb{C}^{2 n}$ and $\mathbb{C}^{2} \otimes \mathbb{C}^{2 n}$, respectively, i.e. let

$$
\left(h_{1}^{o}:=(1,0), h_{2}^{o}:=(0,1)\right), \quad\left(e_{a}^{o}:=(0, \ldots, \underset{a \text {-th entry }}{1}, \ldots, 0)\right), \quad\left(e_{i a}^{o}:=h_{i}^{o} \otimes e_{a}^{o}\right) .
$$

Further, for any $\left(\mathbb{C}\right.$-linearly extended) adapted frame $u:\left(\mathbb{M}^{n}\right)^{\mathbb{C}}=\mathbb{C}^{2} \otimes \mathbb{C}^{2 n} \rightarrow T_{x}^{\mathbb{C}} M$, denote the corresponding bases for $T_{x}^{\complement} M, \mathbf{H}_{x}$ and $\mathbf{E}_{x}$ by

$$
\left(e_{i a}:=u\left(e_{i a}^{o}\right)\right), \quad\left(e_{i}:=u\left(e_{i a}^{o}\right) \quad \bmod \mathrm{SL}_{2 n}(\mathbb{C})\right), \quad\left(e_{a}:=u\left(e_{i a}^{o}\right) \quad \bmod \mathrm{SL}_{2}(\mathbb{C})\right) .
$$

Note that when an adapted frame $u: \mathbb{H}^{n} \rightarrow T_{x} M$ is changed into another adapted frame,

$$
u^{\prime}=u \circ U \quad \text { with } \quad U=\left(\begin{array}{ll}
u_{+}^{1} & u_{-}^{1} \\
u_{+}^{2} & u_{-}^{2}
\end{array}\right) \in \operatorname{Sp}_{1} \subset \mathrm{SL}_{2}(\mathbb{C})=\operatorname{Sp}_{1}(\mathbb{C}),
$$

the corresponding complex basis $\left(e_{j a}\right)$ of $T_{x}^{\mathbb{C}} M$ changes to

$$
e_{+a}=u_{+}^{j} e_{j a}, \quad e_{-a}=u_{-}^{j} e_{j a},
$$

and the corresponding basis $\left(e_{i}\right)$ for the 2-dimensional space $\mathbf{H}_{x} \simeq \mathbb{C}^{2}$ transforms into

$$
e_{+}:=u_{+}^{i} e_{i}, \quad e_{-}:=u_{-}^{i} e_{i} .
$$

We shall frequently use the complex frames $\left(e_{ \pm a}\right)$ and $\left(e_{ \pm}\right)$and for brevity, we call them the adapted complex frames for $T_{x}^{\complement} M$ and $\mathbf{H}_{x}$, respectively.

Note that each adapted complex frame $\left(e_{+}, e_{-}\right)$for $\mathbf{H}_{x} \simeq \mathbb{C}^{2}$ is orthonormal and symplectic and is therefore uniquely determined, up to the sign of the second vector $e_{-}$, by just its first vector $e_{+}$. This means that the projection 


$$
j: \mathcal{C} O_{g}(M, Q) \rightarrow \mathbf{H}^{o}:=\mathbf{H} \backslash\{\text { zero section }\}, j\left(t e_{+a}\right):=t e_{+}, \quad t \in(0,+\infty),
$$

determines a fibre preserving diffeomorphism between the Swann bundle $\pi: \mathcal{S} \rightarrow M$ and the holomorphic bundle $p: \mathbf{H}^{o} \rightarrow M$,

$$
\widetilde{j}: \mathcal{S}=\mathcal{C} O_{g}(M, Q) / \mathrm{Sp}_{p, q} \rightarrow \mathbf{H}^{o} .
$$

We may therefore geometrically identify $\mathcal{S}$ with the bundle $\mathbf{H}^{o} \rightarrow M$, provided that $\mathbf{H}^{o}$ is equipped with the structure of a principal $\mathbb{1}^{*} / \mathbb{Z}_{2}$-bundle determined by the right action of $\mathbb{R}^{*} \times_{\mathbb{Z}_{2}}\left(\operatorname{Sp}_{1} \times_{\mathbb{Z}_{2}} \operatorname{Sp}_{p, q}\right)$ on $\mathcal{C O}_{g}(M, Q)$ through the projection (3.12) of $\mathcal{C O}(M, Q)$ onto $\mathbf{H}^{o}$.

\section{5 $\mathbb{M} P^{n}$ and its hyperkähler cone}

Consider now the quaternionic projective space $\mathbb{H} P^{n}=\left(\mathbb{M}^{n+1} \backslash\{0\}\right) / \mathbb{H}^{*}$, with $\mathbb{H}^{*}$ acting on the right, and the following related notational data.

(1) We denote by $\left\langle q, q^{\prime}\right\rangle=\sum_{i=0}^{n} \bar{q}^{i} q^{i}$ the standard Hermitian product of elements $q, q^{\prime} \in \mathbb{H}^{n+1}$ and by $\left(e_{j}^{o}\right)$ the standard orthonormal basis of $\mathfrak{H}^{n+1} \simeq \mathbb{R}^{4(n+1)}$ with

$$
\begin{aligned}
& e_{4 k+1}^{o}:=(0, \ldots, 0, \underset{(4 k+1) \text {-th place }}{1}, 0 \ldots, 0), \\
& e_{4 k+2}^{o}:=(0, \ldots, 0, \underset{(4 k+1) \text {-th place }}{\mathbf{i}}, 0 \ldots, 0), \\
& e_{4 k+3}^{o}:=(0, \ldots, 0, \underset{(4 k+1) \text {-th place }}{\mathbf{j}}, 0 \ldots, 0), \\
& e_{4 k+4}^{o}:=(0, \ldots, 0, \underset{(4 k+1) \text {-th place }}{\mathbf{k}}, 0 \ldots, 0), \quad \text { with } 0 \leq k \leq n .
\end{aligned}
$$

(2) $S\left(\mathbb{E}^{n+1}\right)$ is the unit sphere of $\mathbb{H}^{n+1}$. The natural projection $\pi: S\left(\mathbb{H}^{n+1}\right) \rightarrow \mathbb{H} P^{n} \simeq S\left(\mathbb{M}^{n+1}\right) / S(\mathbb{M})$ is the well-known Hopf fibration.

(3) $g_{o}$ is the Riemannian metric of $\mathbb{H} P^{n}$ defined by projecting each inner product $\left.\langle\cdot, \cdot\rangle\right|_{\mathcal{D}_{q} \times \mathcal{D}_{q}}$ of the spaces of the distribution $\mathcal{D} \subset T S\left(\mathbb{H}^{n+1}\right)$ orthogonal to the fibres of the Hopf fibration onto the corresponding tangent space $T_{[q]} \mathbb{H} P^{n},[q] \in \mathbb{H} P^{n}$.

(4) $Q \subset$ End $\left(T \llbracket P^{n}\right)$ is the bundle of endomorphisms of the tangent spaces determined as

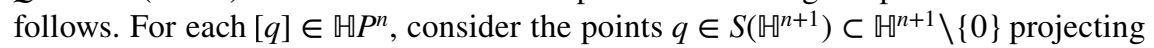
onto $[q]$ and the triples of complex structures on the horizontal spaces $\mathcal{D}_{q} \subset T_{q} S\left(\mathbb{\boxplus}^{n+1}\right)$ determined by the right multiplications by $\mathbf{i}, \mathbf{j}$ and $\mathbf{k}$. Each triple projects onto a different hypercomplex structure, but each such projected hypercomplex structure spans a 3-dimensional vector space $Q_{[q]} \subset$ End $\left(T_{[q]} \mathbb{M} P^{n}\right)$, which is independent of $q \in[q]$. The bundle $Q$ is defined as $Q:=\bigcup_{[q] \in \mathbb{H} P^{n}} Q_{[q]}$.

The triple ( $\left.\mathbb{H} P^{n}, g_{o}, Q\right)$ is a typical example of a homogeneous qk manifold. We remark that the standard isometric left action of $\mathrm{Sp}_{n+1}$ on $\mathbb{W}^{n+1}$ acts transitively and effectively on each sphere $t S\left(\mathbb{H}^{n+1}\right)$ of radius $t>0$ and with isotropy $\mathrm{Sp}_{n}$. This transitive left action commutes with the right action of $S \mathrm{p}_{1}$ and gives rise to the $\mathrm{Sp}_{n+1}$-equivariant map

$$
j^{(t)}: t S\left(\mathbb{M}{ }^{n+1}\right) \simeq \mathrm{Sp}_{n+1} / \mathrm{Sp}_{n} \longrightarrow \mathbb{M} P^{n} \simeq \mathrm{Sp}_{n+1} / \mathrm{Sp}_{n} \times_{\mathbb{Z}_{2}} \mathrm{Sp}_{1}
$$


for each $t \in(0,+\infty)$. The left $\mathrm{Sp}_{n+1}$-action on $\mathbb{H} P^{n}$ is isometric for each homothetic metric $t g_{o}$ and it naturally lifts to the level of linear frames, mapping the bundle of adapted frames into itself. All this allows the identifications

$$
O_{t_{g_{o}}}\left(\mathbb{T}^{n}, Q\right) \simeq \mathrm{Sp}_{n+1} / \mathbb{Z}_{2}, \quad \mathcal{C} O_{g_{o}}\left(\mathbb{T}^{n}, Q\right) \simeq \mathbb{R}_{>0} \times \mathrm{Sp}_{n+1} / \mathbb{Z}_{2}
$$

and shows the existence of an $\mathrm{Sp}_{n+1}$-equivariant diffeomorphism

$$
j: \mathbb{M}^{n+1} \backslash\{0\} / \mathbb{Z}_{2}=\bigcup_{t \in(0,+\infty)} t S\left(\mathbb{M}^{n+1}\right) / \mathbb{Z}_{2} \longrightarrow \mathcal{S}=\mathcal{C} O_{g_{o}}\left(\mathbb{T}^{n}, Q\right) / \mathrm{Sp}_{n}
$$

This implies that the Swann bundle $\pi: \mathcal{S} \rightarrow \mathbb{H} P^{n}$ is equivalent to

$$
p:\left(\mathbb{T}^{n+1} \backslash\{0\}\right) / \mathbb{Z}_{2} \rightarrow \mathbb{H}^{n},
$$

the $\mathbb{Z}_{2}$-quotient of the tautological bundle of $\mathbb{H} P^{n}$. Note that both the flat metric $h_{o}=\langle\cdot, \cdot\rangle$ and the standard hypercomplex structure $(\mathbf{i}, \mathbf{j}, \mathbf{k})$ of $\mathbb{\boxplus}^{n+1} \backslash\{0\}$, given by the right multiplications by the three complex structures of $\mathbb{H}$, are invariant under the left $\mathrm{Sp}_{n+1}$-action. Using this, it can be checked that the right action of $\mathbb{U}^{*}=\mathbb{R}_{>0} \times \mathrm{Sp}_{1}$ on $\left(\mathbb{T}^{n+1} \backslash\{0\}\right) / \mathbb{Z}_{2}$ transforms $h_{o}$ and $(\mathbf{i}, \mathbf{j}, \mathbf{k})$ as prescribed for $\mathbb{U}^{*}$-conical manifolds, so that $\left(\left(\mathbb{Q}^{n+1} \backslash\{0\}\right) / \mathbb{Z}_{2}, h_{o}, \mathbf{i}, \mathbf{j}, \mathbf{k}\right)$ is identifiable with the hk cone of $\left(\mathbb{H} P^{n}, g_{o}, Q\right)$.

\section{Harmonic spaces}

\subsection{Harmonic space of a hyperkähler manifold}

Let $\left(N, g, J_{1}, J_{2}, J_{3}\right)$ be an hk manifold, i.e. a qk manifold admitting a $\nabla$-parallel hypercomplex structure $\left(J_{1}, J_{2}, J_{3}\right)$ that generates the fibre $Q_{x} \subset Q$ at each $x \in N$. Then, for any point $z=(a, b, c) \in \mathbb{R}^{3}$ which is in the unit sphere $S^{2} \simeq \mathbb{C} P^{1}$, the linear combination $I^{(z)}:=a J_{1}+b J_{2}+c J_{3}$ is also a $\nabla$-parallel integrable complex structure. The twistor bundle $\mathcal{Z}(N)$ of $\left(N, g, J_{1}, J_{2}, J_{3}\right)$ is the trivial $\mathbb{C} P^{1}$-bundle over $N$ defined by

$$
\pi^{\mathcal{Z}}: \mathcal{Z}(N):=N \times\left\{I^{(z)}, z \in S^{2}\right\} \simeq N \times \mathbb{C} P^{1} \rightarrow N .
$$

It is naturally equipped with an integrable complex structure $\hat{I}$, coinciding with the complex structures $I^{(z)}$ along the horizontal leaves $N \times\{z\}$, as well as with the standard $\mathrm{SL}_{2}(\mathbb{C})$ -invariant complex structure of $\mathbb{C} P^{1}$ along the vertical spaces $\{x\} \times \mathbb{C} P^{1}[18,26]$.

The harmonic space $\mathcal{H}(N)$ of $\left(N, g, J_{1}, J_{2}, J_{3}\right)$ is an analogous trivial bundle, albeit with larger fibres. It is defined as the trivial $\mathrm{SL}_{2}(\mathbb{C})$-bundle

$$
\pi^{\mathcal{H}}: \mathcal{H}(N):=N \times \mathrm{SL}_{2}(\mathbb{C}) \rightarrow N
$$

equipped with the following integrable complex structure. For each $(x, U) \in \mathcal{H}(N)$, consider the direct sum decomposition $T_{(x, U)} \mathcal{H}(N)=T_{x} N+T_{U} \mathrm{SL}_{2}(\mathbb{C}) \simeq T_{x} N+\mathfrak{s l}_{2}(\mathbb{C})$, where $T_{U} \mathrm{SL}_{2}(\mathbb{C})$ is identified with $\mathfrak{S l}_{2}(\mathbb{C})$ by using right invariant vector fields $\left(^{1}\right)$. Now, denote by $\rrbracket_{(x, U)}$ the unique linear map on $T_{(x, U)} \mathcal{H}(N)$ satisfying

\footnotetext{
${ }^{1}$ More precisely, we assume that any $v \in T_{\downarrow} \mathrm{SL}_{2}(\mathbb{C})$ is identified with the unique element $E^{(v)} \in \mathfrak{s} \mathfrak{I}_{2}(\mathbb{C})$, whose associated right invariant vector field $\widehat{E^{(v)}}$ is such that $\left.\widehat{E^{(v)}}\right|_{U}=v$.
} 


$$
\left.\rrbracket_{(x, U)}\right|_{T_{x} N}:=\left.I^{(z)}\right|_{x}, \quad z=U \cdot[0: 1] \in \mathbb{C} P^{1},\left.\quad \rrbracket_{(x, U)}\right|_{\mathfrak{s} \mathfrak{L}_{2}(\mathbb{C})}=J_{o},
$$

where $J_{o}$ is the complex structure of $\mathfrak{g l}_{2}(\mathbb{C})$ given by the multiplication by $\left(\begin{array}{ll}i & 0 \\ 0 & i\end{array}\right)$. The collection of linear maps $\rrbracket_{(x, U)}$ defines an almost complex structure $\llbracket$ on $\mathcal{H}(N)$, which can be checked to be integrable. The complex manifold $(\mathcal{H}(N), \mathbb{0})$ naturally projects to the twistor space $(\mathcal{Z}(N), \widehat{I})$, the projection being holomorphic with fibres given by the orbits in $\mathcal{H}(N)$ of the subgroup $B \subset \mathrm{SL}_{2}(\mathbb{C})$ of upper triangular matrices (see, e.g. [13, §3.2]).

\subsection{Harmonic space of a quaternionic Kähler manifold}

We now proceed to the harmonic space of a strict qk manifold. One might expect this to be defined analogously to the hyperkähler case: The harmonic space $\mathcal{H}(N)$ of an hk manifold $N$ is a topologically trivial bundle equipped with a non-product complex structure which makes it a holomorphic bundle over its twistor space $Z(N)$. Now, the twistor construction for any qk manifold was introduced by Salamon [26] regardless of the sign of its scalar curvature as a $\mathbb{C} P^{1}$-bundle in analogy with the Penrose construction in real dimension four. As a matter of fact it applies equally well in the more general context of quaternionic manifolds, see [2] for example. However, this twistor space does not serve as a suitable base for a prospective harmonic space for a strict qk manifold $M$, having the drawback that the lifted gauge field is not partially flat in the sense required for the application of the harmonic space method (see, e.g. [15]). This difficulty can be circumvented by considering instead of the qk manifold its hyperkähler cone. We thus have:

Definition 4.1 The harmonic space of a (strict) qk manifold $(M, g, Q)$ is the bundle over $M$ given by the pair $\left(\mathcal{H}(M)^{\mathrm{qk}}, \pi^{\mathcal{H}^{\mathrm{qk}}}\right)$, where

(i) the total space $\mathcal{H}(M)^{\mathrm{qk}}:=\mathcal{H}(\mathcal{S})$ is the harmonic space of the hk cone $\left(\mathscr{S}, h, \mathbb{J}_{\alpha}\right)$ of $M$ (cf. (4.1)) and

(ii) the projection $\pi^{\mathrm{qk}}$ is the map

$\pi^{\mathrm{qk}}=\pi \circ \pi^{\mathcal{H} \mid \mathcal{S}}: \mathcal{H}(M)^{\mathrm{qk}}=\mathcal{S} \times \mathrm{SL}_{2}(\mathbb{C}) \rightarrow M$,

determined by composing the projection $\pi^{\mathcal{H} \mid \mathcal{S}}: \mathcal{H}(M)^{\mathrm{qk}}=\mathcal{H}(\mathcal{S}) \rightarrow \mathcal{S}$ of the harmonic space $\mathcal{H}(\mathcal{S})$ onto $\mathcal{S}$ with the projection $\pi: \mathcal{S} \rightarrow M$ of the Swann bundle over $M$.

In other words, $\mathcal{H}(M)^{\mathrm{qk}}$ is precisely the harmonic space $\mathcal{H}(M)^{\mathrm{qk}}=\mathcal{H}(\mathcal{S})$ of $\mathcal{S}$, considered as a bundle over $M$ rather than one over $\mathcal{S}$.

The following lemma gives a few basic properties of $\mathcal{H}(M)^{\mathrm{qk}}$.

Lemma 4.2 The manifold $\mathcal{H}(M)^{\mathrm{qk}}$ has a natural integrable complex structure and is acted on holomorphically and freely by the group

$$
L:=\left(\mathbb{W}^{*} / \mathbb{Z}_{2}\right) \ltimes \mathrm{SL}_{2}(\mathbb{C}) .
$$

The action is transitive on the fibres of the projection $\pi^{\mathrm{qk}}: \mathcal{H}(M)^{\mathrm{qk}} \rightarrow M$ and gives $\mathcal{H}(M)^{\mathrm{qk}}$ the structure of a principal L-bundle over $M$. 
Proof Since $\mathcal{H}(M)^{\mathrm{qk}}$ is the harmonic space of an hk manifold, the discussion in [13, §3.2] implies that it is naturally equipped with an integrable complex structure with respect to which the orbits of the right action of $\mathrm{SL}_{2}(\mathbb{C})$ are complex. Further, $\mathcal{H}(M)^{\mathrm{qk}}$ can be (locally) identified with the bundle of adapted complex frames $\left(e_{+}, e_{-}\right)$for the rank 2 holomorphic vector bundle $\mathbf{H} \rightarrow \mathcal{S}$ defined in Sect. 3.4. Now, for any element $a \in \mathbb{H}^{*} / \mathbb{Z}_{2}$, let us consider the corresponding diffeomorphism $f_{a}: \mathcal{S} \rightarrow \mathcal{S}$ determined by the right action of $\mathbb{U}^{*} / \mathbb{Z}_{2}$ on $\mathcal{S}$ described in detail in Sect. 3.2. From this description, it follows immediately that the push-forward $f_{a *}: T \mathcal{S} \rightarrow T \mathcal{S}$ transforms any complex adapted frame of $\left(\mathcal{S}, h, J_{\alpha}\right)$ into another complex adapted frame. This means that the push-forward map $f_{a *}$ determines an automorphism of the bundle of the vertical frames $\left(e_{+}, e_{-}\right)$of $\mathbf{H}$ which preserves its complex structure. This is tantamount to saying that each element $a \in \mathbb{U}^{*} / \mathbb{Z}_{2}$ is uniquely associated with a fibre preserving biholomorphism of $\pi^{\mathcal{H} \mid \mathcal{S}}: \mathcal{H}(M)^{\mathrm{qk}} \rightarrow \mathcal{S}$. These biholomorphisms together with those of the $\mathrm{SL}_{2}(\mathbb{C})$-action determine an effective right action of $L=\left(\mathbb{\boxplus}^{*} / \mathbb{Z}_{2}\right) \ltimes \mathrm{SL}_{2}(\mathbb{C})$ on $\mathcal{H}(M)^{\mathrm{qk}}$ acting transitively on the fibres of $\mathcal{H}(M)^{\mathrm{qk}}$ over $M$.

Remark 4.3 We recall that $\mathcal{H}(M)^{\mathrm{qk}}$, although equipped with a non-product complex structure, is topologically a cartesian product of $\mathrm{SL}_{2}(\mathbb{C})$ and $\mathcal{S}$. Now, $\mathcal{S}$ is an $\mathbb{U}^{*} / \mathbb{Z}_{2}$-bundle over $M$. So the product group $\mathrm{SL}_{2}(\mathbb{C}) \times\left(\mathbb{H}^{*} / \mathbb{Z}_{2}\right)$ acts naturally and transitively on the fibres of $\pi^{\mathrm{qk}}: \mathcal{H}(M)^{\mathrm{qk}} \rightarrow M$. This natural action restricts to an action of the $\mathbb{Q}^{*} / \mathbb{Z}_{2}$ subgroup on $\mathcal{H}(M)^{\mathrm{qk}}$, which leaves each horizontal leaf $\mathcal{S} \times\{U\} \subset \mathcal{S} \times \mathrm{SL}_{2}(\mathbb{C})=\mathcal{H}(M)^{\mathrm{qk}}$ invariant. However, this action of $\mathbb{U}^{*} / \mathbb{Z}_{2}$ is not holomorphic. In contrast, the action of the semi-direct product $L=\left(\mathbb{Q}^{*} / \mathbb{Z}_{2}\right) \ltimes \mathrm{SL}_{2}(\mathbb{C})$ described in the above proof is holomorphic and hence restricts to a new action of its subgroup $\mathbb{U}^{*} / \mathbb{Z}_{2}$ which is also holomorphic. The price we have to pay is that the horizontal leaves $\mathcal{S} \times\{U\}$ are no longer invariant under this action. More precisely, an element $a=t q \bmod \mathbb{Z}_{2}$ with $t \in \mathbb{R}_{>0}, q \in \mathrm{Sp}_{1}$, of the group $\mathbb{U}^{*} / \mathbb{Z}_{2}$ biholomorphically maps each leaf $\mathcal{S} \times\{U\}$ (identified with a vertical frame field $\left(e_{ \pm}\right)$of $\mathbf{H}$ by means of a fixed local equivalence $T^{\mathbb{C}} M \simeq \mathbf{H} \otimes \mathbf{E}$ ) into the new leaf $\mathcal{S} \times\left\{U^{\prime}=\operatorname{Ad}_{q}(U)\right\}$ (identified with the vertical frame field $\left(R_{q_{*}}\left(e_{ \pm}\right)\right)$under the same local equivalence).

From the discussion in Sect. 3.5, the harmonic space $\mathcal{H}(M)^{\mathrm{qk}}$ of $M=\mathbb{H} P^{n}$ is identifiable with the bundle

$$
\pi^{\mathrm{qk}}:\left(\mathbb{M}^{n+1} \backslash\{0\} / \mathbb{Z}_{2}\right) \times \mathrm{SL}_{2}(\mathbb{C}) \rightarrow \mathbb{M} P^{n}=\left(\mathbb{M}^{n+1} \backslash\{0\}\right) / \mathbb{M}^{*} .
$$

Note that this $\mathcal{H}(M)^{\mathrm{qk}}$ can be seen as a principal bundle with respect to both the action of the direct product $\left.\left(\mathbb{E}^{n+1} \backslash\{0\} / \mathbb{Z}_{2}\right) \times \mathrm{SL}_{2}(\mathbb{C})\right)$, as well as the action of the semi-direct product $\left.L=\left(\mathbb{M}^{n+1} \backslash\{0\} / \mathbb{Z}_{2}\right) \ltimes \mathrm{SL}_{2}(\mathbb{C})\right)$, depending on whether $\mathcal{H}(M)^{\mathrm{qk}}$ is considered as a cartesian product or as a quotient of an appropriate bundle of linear frames.

\subsection{Complexified harmonic space}

Consider an $n$-dimensional complex manifold $(N, J)$. The complexification of $(N, J)$ is the pair $\left(N^{\mathbb{C}}, \imath\right)$ given by:

(a) the complex manifold $N^{\mathbb{C}}:=N \times N$ having complex structure $\widetilde{J}$ defined at each point $(x, y) \in N \times N$ by $\widetilde{J}_{(x, y)}(v, w):=J_{x}(v)-J_{y}(w)$

(b) the standard diagonal embedding $\imath: N \rightarrow N^{\mathbb{C}}, \imath(x)=(x, x)$. 
Note that $\widetilde{J}$ is so defined that the complete atlas of holomorphic coordinates of $\left(N^{\mathbb{C}}, \widetilde{J}\right)$ is precisely the one generated by the collection of complex coordinates of the form $\widetilde{\xi}=\left(z^{i}, \overline{z^{\prime j}}\right): \mathcal{U} \times \mathcal{V} \rightarrow \mathbb{C}^{2 n}$ determined by pairs of holomorphic coordinates $\left(z^{i}\right),\left(z^{\prime j}\right)$ of $(N, J)$.

This notion of "complexification" of a complex manifold yields the following notion of "complexified harmonic space". Consider the harmonic space $\mathcal{H}(M)^{\mathrm{qk}}=\mathcal{S} \times \mathrm{SL}_{2}(\mathbb{C})$ of a (strict) qk manifold $M$ and denote by $\mathcal{H}^{\mathbb{C}}(M)^{\mathrm{qk}}$ the cartesian product $\mathcal{H}^{\mathbb{C}}(M)^{\mathrm{qk}}=\mathcal{S} \times \mathcal{S} \times \mathrm{SL}_{2}(\mathbb{C})$ equipped with the unique (integrable) complex structure $\rrbracket^{(\mathbb{C})}$ which coincides with the right invariant complex structure along the (vertical) leaves $\{x\} \times\{y\} \times \mathrm{SL}_{2}(\mathbb{C}) \simeq \mathrm{SL}_{2}(\mathbb{C})($ see (4.2)) and with the complex structure of the complexification of $\left(\mathcal{S}, I^{(z)}\right), z=U \cdot[0: 1]$, along each horizontal leaf $\mathcal{S} \times \mathcal{S} \times\{U\}$. In other words, $\mathcal{H}^{\mathbb{C}}(M)^{\mathrm{qk}}$ is the union of the complexifications of the manifolds $\left(\mathcal{S}, I^{(z)}\right), z \in S^{2}$. This is clearly not identical to a "complexification" of $\left(\mathcal{H}(M)^{\mathrm{qk}}, \square\right)$ in the sense of the previous paragraph; rather it is merely a partial complexification in that context. Nevertheless, for the sake of brevity, we simply call it the complexified harmonic space.

\section{Prepotentials for instantons on a qk manifold}

\subsection{Lifts of instantons}

Let $(E, D)$ be a gauge field on a (strict) qk manifold $(M, g, Q)$, associated with the pair $(P, \omega)$, a principal bundle $P$ over $M$ with a connection $\omega$. Further, the lift $\left(P^{\prime}, \omega^{\prime}\right)$ of $(P, \omega)$ to the harmonic space $\pi^{\mathrm{qk}}: \mathcal{H}(M)^{\mathrm{qk}} \rightarrow M$ is the pair given by the lifted bundle $P^{\prime}=\pi^{\mathrm{qk} *} P$ over $\mathcal{H}(M)^{\mathrm{qk}}$ together with the natural lift $\omega^{\prime}$ to $P^{\prime}$ of $\omega$ (see, e.g. [13, §2.4]). The lift of $(E, D)$ to $\mathcal{H}(M)^{\mathrm{qk}}$ is the gauge field $\left(E^{\prime}=\pi^{\mathrm{qk} *} E, D^{\prime}=\pi^{\mathrm{qk} *} D\right)$ given by

(i) the lifted vector bundle $q^{\prime}: E^{\prime}=\pi^{\mathrm{qk} *} E=P^{\prime} \times_{G, \rho} V \rightarrow \mathcal{H}(M)^{\mathrm{qk}}$ and

(ii) the covariant derivative $D^{\prime}=\pi^{\mathrm{qk} *} D$ on $E^{\prime}$ determined by $\omega^{\prime}$.

Proposition 5.1 Let $\left(E^{\prime}, D^{\prime}\right)$ be a gauge field associated with a principal bundle with connection $\left(P^{\prime}, \omega^{\prime}\right)$ on $\mathcal{H}(M)^{\mathrm{qk}}$. Then, $\left(E^{\prime}, D^{\prime}\right)$ is the lift of an instanton on $M$ if and only if:

(a) The $\omega^{\prime}$-horizontal vector fields on $P^{\prime}$ of the infinitesimal transformations of the right $L$-action on $\mathcal{H}(M)^{\mathrm{qk}}$ are complete and the group action generated by these vector fields has no singular orbit.

(b) The curvature $F^{\prime}$ of $D^{\prime}$ is such that $F^{\prime}(E, \cdot)=0$ for any infinitesimal transformation $E$ of the L-action.

(c) For any $4 n$-tuple $\left(e_{ \pm a}^{\prime}\right)$ of vectors in the complex tangent spaces $T_{x}^{\mathbb{C}} \mathcal{H}(M)^{\mathrm{qk}}$, which project onto an adapted complex frame $\left(e_{+a}, e_{-b}\right)$ of $M$, the following relations hold

$F^{\prime}\left(e_{+a}^{\prime}, e_{+b}^{\prime}\right)=F^{\prime}\left(e_{-a}^{\prime}, e_{-b}^{\prime}\right)=0, \quad F^{\prime}\left(e_{+a}^{\prime}, e_{-b}^{\prime}\right)=-F^{\prime}\left(e_{-a}^{\prime}, e_{+b}^{\prime}\right)$.

Proof By [13, Prop. 2.2], conditions (a) and (b) hold if and only if $\left(E^{\prime}, D^{\prime}\right)$ is the lift of a gauge field $(E, D)$ on $M$. Such a gauge field is an instanton if and only if the component $F^{(2)}$ 
of its curvature, defined in (2.8), is identically zero. This holds if and only if (c) holds.

Corollary 5.2 The lift $\left(E^{\mathcal{S}}, D^{\mathcal{S}}\right)$ to the Swann bundle $\mathcal{S}$ of an instanton $(E, D)$ on $(M, g, Q)$ is an instanton on the hyperkähler cone $\left(\mathcal{S}, h, \mathbb{J}_{\alpha}\right)$.

If, in addition, the instanton $(E, D)$ is the complexification of an instanton with compact structure group $G^{o}$, its lift $\left(E^{\prime}, D^{\prime}\right)$ to $\mathcal{H}(M)^{\mathrm{qk}}$ is real analytic and admits local holomorphic extensions on open sets of $\mathcal{H}^{\mathbb{C}}(M)^{\mathrm{qk}}$.

Proof We recall that $\mathcal{H}(M)^{\mathrm{qk}}$ is the harmonic space of the hk manifold $\left(\mathcal{S}, h, \mathbb{J}_{\alpha}\right)$, so the lift $\left(E^{\prime}, D^{\prime}\right)$ to $\mathcal{H}(M)^{\mathrm{qk}}$ can also be considered as the lift of a gauge field $\left(E^{\mathcal{S}}, D^{\mathcal{S}}\right)$ on the hk manifold $\mathcal{S}$. The metric $h$ and the complex structures $\rrbracket_{\alpha}$ of $\mathcal{S}$ imply the existence of an adapted complex frame $\left(e_{+a}^{\mathcal{S}}, e_{-b}^{\mathcal{S}}\right)$ for the complexified tangent spaces $T_{y}^{\mathbb{C}} \mathcal{S}$, consisting of

(i) four complex vectors, say $e_{ \pm 1}^{\mathcal{S}}, e_{ \pm 2}^{\mathcal{S}}$, vertical with respect to the projection onto $M$; in particular, each of them is a linear combination of the projections from $\mathcal{H}(M)^{\mathrm{qk}}$ onto $\mathcal{S}$ of infinitesimal holomorphic transformations of $L$, and

(ii) additional $4 n$ complex vectors $e_{ \pm a}^{\mathcal{S}}, a \geq 3$, projecting onto the elements of an adapted complex frame $\left(e_{ \pm(a-2)}\right)$ of a qk manifold $(M, t \cdot g, Q)$ homothetic to $(M, g, Q)$.

Since the curvature $F^{\prime}$ of $\left(E^{\prime}, D^{\prime}\right)$ satisfies Proposition 5.1 and $\left(E^{\prime}, D^{\prime}\right)$ is the lift of the gauge field $\left(E^{\mathcal{S}}, D^{\mathcal{S}}\right)$, (i) and (ii) imply that $\left(E^{\prime}, D^{\prime}\right)$ satisfies all the hypotheses of [13, Lemma 4.2] with respect to the base manifold $\mathcal{S}$. This implies that $\left(E^{\mathcal{S}}, D^{\mathcal{S}}\right)$ is an instanton on $\mathcal{S}$. The final claim of the corollary follows from [13, Prop. 4.1] and the fact that $\left(E^{\prime}, D^{\prime}\right)$ is the lift of $\left(E^{\mathcal{S}}, D^{\mathcal{S}}\right)$.

\subsection{Adapted coordinates and adapted frames on harmonic space}

\subsubsection{Holomorphically adapted coordinates}

Let $x_{o} \in M$ be a point of a qk manifold and $\mathcal{U}$ one of its neighbourhoods on which there exist trivialisations of the bundle $\pi: \mathcal{S} \simeq \mathbf{H}^{o} \rightarrow M$ allowing the identifications $\left.\mathcal{S}\right|_{\mathcal{U}} \simeq \mathcal{U} \times \mathbb{M}^{*}$. We may write this as $\left.\mathcal{S}\right|_{\mathcal{U}} \simeq \mathcal{U} \times\left(\mathbb{C}^{2} \backslash\{0\}\right)$, representing quaternions $q=x^{0}+x^{1} \mathbf{i}+x^{2} \mathbf{j}+x^{3} \mathbf{k}$ as complex pairs, $q=\left(\eta=x^{0}+\mathbf{i} x^{1}, \zeta=x^{2}+\mathbf{i} x^{3}\right)$. In this way, any set of coordinates $\left(x^{i}\right): \mathcal{U} \subset M \rightarrow \mathbb{R}^{4 n}$ on $\mathcal{U}$ induces corresponding coordinates on $\left.\mathcal{S}\right|_{\mathcal{U}}$,

$$
\widehat{\xi}=\left(x^{1}, \ldots, x^{4 n} ; \eta, \zeta\right):\left.\mathbf{H}^{o}\right|_{\mathcal{U}} \longrightarrow \mathbb{R}^{4 n} \times\left(\mathbb{C}^{2} \backslash\{0\}\right), \quad(\eta, \zeta) \neq(0,0) .
$$

We call such a system of coordinates adapted coordinates for $\mathcal{S}$. Now, **denoting by $u_{ \pm}^{i}$ the elements of the matrices $U=\left(\begin{array}{ll}u_{+}^{1} & u_{-}^{1} \\ u_{+}^{2} & u_{-}^{2}\end{array}\right)$ in $\mathrm{SL}_{2}(\mathbb{C})$, each element of the $L$-invariant open set $\left.\mathcal{H}(M)^{\mathrm{qk}}\right|_{\mathcal{U}}=\left(\pi^{\mathrm{qk}}\right)^{-1}(\mathcal{U}) \simeq \mathcal{U} \times\left(\mathbb{C}^{2} \backslash\{0\}\right) \times \mathrm{SL}_{2}(\mathbb{C})$ is uniquely determined by a tuple of $4 n$ real and 6 complex numbers

$$
\left(x^{1}, \ldots, x^{4 n}, \eta, \zeta, u_{ \pm}^{i}\right) \quad \text { constrained by } \quad(\eta, \zeta) \neq(0,0), \operatorname{det}\left(u_{ \pm}^{i}\right)=1 .
$$


They do not form a true set of coordinates because they are not functionally independent. However, they are very convenient and, with a minor abuse of language, we call them the adapted coordinates of $\mathcal{H}(M)^{\mathrm{qk}}$.

Finally, consider the complex structure $I^{\left(z_{o}\right)}, z_{o}=[0: 1]$, of the horizontal leaf $\left.\mathcal{S}\right|_{\mathcal{U}} \times\left\{I_{2}\right\} \subset \mathcal{H}(M)^{\mathrm{qk}}$ (for the definition of $I^{(z)}$, see Sect. 4.1) and denote by $\left(z^{j a}\right), j=1,2$, $1 \leq a \leq 2 n+2$, a set of coordinates on the complexification $\left.\mathcal{S}\right|_{\mathcal{U}} \times\left.\mathcal{S}\right|_{\mathcal{U}}$ of the complex manifold $\left(\left.\left.\mathcal{S}\right|_{\mathcal{U}} \simeq \mathcal{S}\right|_{\mathcal{U}} \times\left\{I_{2}\right\}, I^{\left(z_{o}\right)}\right)$ satisfying the following conditions:

(1) $z^{11}=\eta, z^{12}=\zeta$ and $z^{21}=\bar{\eta}, z^{22}=\bar{\zeta}$.

(2) The coordinates $z^{1 a}, a \geq 3$, and the $z^{11}$ and $z^{12}$ restrict to a set of $I^{\left(z_{o}\right)}$-holomorphic coordinates on $\left.\imath\left(\left.\mathcal{S}\right|_{\mathcal{U}}\right) \subset \mathcal{S}\right|_{U} \times\left.\mathcal{S}\right|_{\mathcal{U}}$.

(3) The coordinates $z^{2 a}$ with $a \geq 3$ restrict to the complex conjugates $z^{2 a}=\overline{z^{1 a}}$ on $\imath\left(\left.\mathcal{S}\right|_{\mathcal{U}}\right)$.

For the remainder of $\left.\mathcal{H}(M)^{\mathrm{qk}}\right|_{\mathcal{U}}=\left.\mathcal{S}\right|_{\mathcal{U}} \times \mathrm{SL}_{2}(\mathbb{C})$, i.e. the points with adapted coordinates of the form $\left(x^{i}, \eta, \zeta, u_{ \pm}^{i}\right)$ with $U=\left(u_{ \pm}^{i}\right) \neq I_{2}$, we choose the $(4 n+8)$-tuple of complex coordinates $\left(z^{+a}, z^{-b}, u_{ \pm}^{i}\right)$ with

$$
z^{+a}:=u_{j}^{+} z^{j a}(x, \eta, \zeta), \quad z^{-a}:=u_{j}^{-} z^{j a}(x, \eta, \zeta)
$$

Here, $u_{j}^{ \pm}$are the entries of the inverse matrix $\left(u_{j}^{ \pm}\right)=\left(u_{ \pm}^{j}\right)^{-1}$, i.e. $u_{j}^{ \pm}= \pm \epsilon_{j k} u_{\mp}^{k}$ with $\left(\epsilon_{j k}\right)=\left(\begin{array}{cc}0 & -1 \\ 1 & 0\end{array}\right)$. Note that:

(a) For any given $U=\left(u_{ \pm}^{i}\right) \in \mathrm{SL}_{2}(\mathbb{C})$, the restrictions of the $z^{+a}$ (resp. $z^{-a}$ ) to the horizontal leaf $\left.\mathcal{S}\right|_{\mathcal{U}} \times\{U\}^{ \pm} \subset \mathcal{H}(M)^{\mathrm{qk}}$ are holomorphic (resp. anti-holomorphic) coordinates with respect to the complex structure $I^{\left(z^{\prime}\right)}$ corresponding to the point $z^{\prime}=U \cdot[0: 1] \in \mathbb{C} P^{1} \simeq S^{2}$. If the $z^{ \pm a}$ are considered to be independent, they are holomorphic coordinates for the complexification of the complex manifold $\left(\left.\mathcal{S}\right|_{\mathcal{U}} \times\{U\}, I^{(z)}\right)$.

(b) At each point, the vector fields $\frac{\partial}{\partial z^{ \pm 1}}, \frac{\partial}{\partial z^{ \pm 2}}$ span the complexified tangent space of the fibre $\left.\mathcal{S}\right|_{x}$ of the Swann bundle over $x \in \mathcal{U} \subset M$.

We call the tuples $\left(z^{ \pm a} u_{ \pm}^{i}\right)$ holomorphically adapted coordinates of $\mathcal{H}^{\mathbb{C}}(M)^{\mathrm{qk}}$.

\subsubsection{Infinitesimal L-transformations in holomorphically adapted coordinates}

Let $\mathfrak{l}=\operatorname{Lie}(L)=\left(\mathbb{R}+\mathfrak{s i t}_{2}\right) \ltimes \mathfrak{s l}_{2}(\mathbb{C})$ and denote by $\mathcal{B}$ the real basis for $\mathfrak{l}$,

$$
\mathcal{B}:=\left(G_{D}^{o}, G_{0}^{o}, G_{1}^{o}, G_{2}^{o}, H_{0}^{o}, H_{++}^{o}, H_{--}^{o}, i H_{0}^{o}, i H_{++}^{o}, i H_{--}^{o}\right) \quad \text { where }
$$

- $G_{D}^{o}$ is the generator of the abelian subgroup $\left\{e^{t}, t \in \mathbb{R}\right\} \subset \mathbb{Q}^{*}$;

- $\left(G_{0}^{o}, G_{1}^{o}, G_{2}^{o}\right)$ is the basis of $\mathfrak{g}_{2}$, given by

$$
G_{0}^{o}:=\left(\begin{array}{cc}
i & 0 \\
0 & -i
\end{array}\right), \quad G_{1}^{o}:=\left(\begin{array}{cc}
0 & 1 \\
-1 & 0
\end{array}\right), \quad G_{2}^{o}:=\left(\begin{array}{cc}
0 & i \\
i & 0
\end{array}\right) ;
$$

- $\left(H_{0}^{o}, H_{++}^{o}, H_{--}^{o}\right)$ is the (complex) basis of $\mathfrak{g l}_{2}(\mathbb{C})$, given by 


$$
H_{0}^{o}:=\left(\begin{array}{cc}
1 & 0 \\
0 & -1
\end{array}\right), \quad H_{++}^{o}:=\left(\begin{array}{ll}
0 & 1 \\
0 & 0
\end{array}\right), \quad H_{--}^{o}:=\left(\begin{array}{ll}
0 & 0 \\
1 & 0
\end{array}\right) .
$$

Now, the isomorphism $\mathbb{U}^{*} / \mathbb{Z}_{2} \simeq \mathbb{R}^{*} \times_{\mathbb{Z}_{2}}\left(\mathrm{Sp}_{1} \times_{\mathbb{Z}_{2}} \mathrm{Sp}_{p, q}\right) / \mathrm{Sp}_{p, q}$ in Sect. 3.2 yields a Lie algebra isomorphism

$$
\mathbb{H}=\operatorname{Lie}\left(\mathbb{Q}^{*} / \mathbb{Z}_{2}\right) \simeq \mathbb{R}+\mathfrak{s} \mathfrak{u}_{2}(\mathbb{C})=\mathfrak{l} / \mathfrak{s l}_{2}(\mathbb{C}),
$$

according to which the unit quaternions $1, \mathbf{i}, \mathbf{j}, \mathbf{k}$ of $\mathbb{H}$ correspond to (the equivalence classes of) $G_{D}^{o}, G_{0}^{o}, G_{1}^{o}$ and $G_{2}^{o}$, respectively.

For each element $G_{\alpha}^{o}$ or $H_{\beta}^{o}$ of $\mathcal{B}$, we denote by $G_{\alpha}$ or $H_{\beta}$ the corresponding holomorphic vector field on $\mathcal{H}(M)^{\mathrm{qk}}$, given by the holomorphic part of the infinitesimal transformation of the corresponding Lie algebra element. The explicit expressions in adapted coordinates of the holomorphic vector fields $G_{\alpha}$ and $H_{\beta}$ can be determined on the basis of the diffeomorphism (3.13) and the description of the corresponding right actions, given in Sect. 3. We obtain

$$
\begin{aligned}
& H_{0}=u_{+}^{i} \frac{\partial}{\partial u_{+}^{i}}-u_{-}^{i} \frac{\partial}{\partial u_{-}^{i}}, \quad H_{++}=u_{+}^{i} \frac{\partial}{\partial u_{-}^{i}}, \quad H_{--}=u_{-}^{i} \frac{\partial}{\partial u_{+}^{i}}, \\
& G_{D}=\eta \frac{\partial}{\partial \eta}+\zeta \frac{\partial}{\partial \zeta}, \quad G_{0}=i \eta \frac{\partial}{\partial \eta}+i \zeta \frac{\partial}{\partial \zeta}+u_{+}^{i} \frac{\partial}{\partial u_{+}^{i}}-u_{-}^{i} \frac{\partial}{\partial u_{-}^{i}}, \\
& G_{1}=-\zeta \frac{\partial}{\partial \eta}+u_{+}^{i} \frac{\partial}{\partial u_{-}^{i}}-u_{-}^{i} \frac{\partial}{\partial u_{+}^{i}}, \quad G_{2}=i \zeta \frac{\partial}{\partial \eta}+i u_{+}^{i} \frac{\partial}{\partial u_{-}^{i}}+i u_{-}^{i} \frac{\partial}{\partial u_{+}^{i}} .
\end{aligned}
$$

The Lie brackets amongst these vector fields are then easily computable:

$$
\begin{array}{lll}
{\left[G_{0}, G_{1}\right]=G_{2},} & {\left[G_{0}, G_{2}\right]=-G_{1},} & {\left[G_{1}, G_{2}\right]=G_{0},} \\
{\left[H_{0}, H_{++}\right]=2 H_{++},} & {\left[H_{0}, H_{--}\right]=-2 H_{--},} & {\left[H_{++}, H_{--}\right]=H_{0},} \\
{\left[G_{0}, H_{0}\right]=0,} & {\left[G_{0}, H_{++}\right]=2 i H_{++},} & {\left[G_{0}, H_{--}\right]= \pm 2 i H_{--},} \\
{\left[G_{1}, H_{0}\right]=-2\left(H_{++}+H_{--}\right),} & {\left[G_{1}, H_{++}\right]=H_{0},} & {\left[G_{1}, H_{--}\right]=H_{0},} \\
{\left[G_{2}, H_{0}\right]=-2 i\left(H_{++}-H_{--}\right),} & {\left[G_{2}, H_{++}\right]=-i H_{0},} & {\left[G_{2}, H_{--}\right]=i H_{0},} \\
{\left[G_{D}, G_{\alpha}\right]=0, \alpha=0,1,2,} & {\left[G_{D}, H_{\beta}\right]=0, \beta \in\{0, \pm \pm\} .} &
\end{array}
$$

\subsubsection{Strongly adapted frames of $\left(\mathcal{S}, h, \rrbracket_{\alpha}\right)$}

In the above collection of holomorphic vector fields, the $H_{\beta}$ are vertical with respect to $\pi^{\mathcal{H} \mid \mathcal{S}}: \mathcal{H}(M)^{\mathrm{qk}} \rightarrow \mathcal{S}$, whereas the $G_{A}$ uniquely decompose as sums $G_{A}=G_{A}^{\mathcal{S}}+V_{A}$, with $V_{A}$ vertical and $G_{A}^{\mathcal{S}}$ tangential to the horizontal leaves $\mathcal{S} \times\{U\} \subset \mathcal{H}(M)^{\mathrm{qk}}$. This decomposition of the $G_{A}$ can be explicitly determined by examining (5.7). There, we can also see that, denoting by $\kappa$ the constant $\kappa=|\Lambda|$ occurring in the definition (3.7) of the hyperkähler metric $h$, the vector fields

$$
\begin{aligned}
& f_{11}:=\frac{1}{2 \kappa\left(|\eta|^{2}+|\zeta|^{2}\right)^{\frac{1}{2}}}\left(G_{D}^{\mathcal{S}}-i G_{0}^{\mathcal{S}}\right)=\frac{1}{\kappa\left(|\eta|^{2}+|\zeta|^{2}\right)^{\frac{1}{2}}}\left(\eta \frac{\partial}{\partial \eta}+\zeta \frac{\partial}{\partial \zeta}\right), \\
& f_{12}:=\frac{1}{2 \kappa\left(|\eta|^{2}+|\zeta|^{2}\right)^{\frac{1}{2}}}\left(G_{1}^{\mathcal{S}}-i G_{2}^{\mathcal{S}}\right)=\frac{1}{\kappa\left(|\eta|^{2}+|\zeta|^{2}\right)^{\frac{1}{2}}}\left(-\zeta \frac{\partial}{\partial \eta}+\eta \frac{\partial}{\partial \zeta}\right)
\end{aligned}
$$


give $h$-orthonormal frames at each vertical tangent space fibre $T^{V} \mathcal{S}_{x}, x \in M$, of the Swann bundle.

We recall that the hyperkähler structure $\left(\mathbb{J}_{1}, \mathbb{J}_{2}, \mathbb{J}_{3}\right)$ of $\mathcal{S}$ acts on vectors as the infinitesimal transformations of $\mathbb{W}=\operatorname{Lie}\left(\mathbb{M}^{*} / \mathbb{Z}_{2}\right)$ associated with the elements $\mathbf{i}, \mathbf{j}, \mathbf{k} \in \mathbb{M}$. Thus, by the identification (5.6), we have

$$
\mathbb{J}_{1} G_{D}^{S}=G_{0}^{\mathcal{S}}, \quad \mathbb{J}_{2} G_{D}^{S}=G_{1}^{\mathcal{S}}, \quad \mathbb{J}_{3} G_{D}=G_{2}^{\mathcal{S}}
$$

and now setting $f_{2 j}:=\overline{f_{1 j}}, j=1,2$, we see that

$$
\mathbb{J}_{1} f_{1 j}=i f_{1 j}, \quad \mathbb{J}_{1} f_{2 j}=-i f_{2 j}, \quad \mathbb{J}_{2} f_{11}=f_{22}, \quad \mathbb{J}_{2} f_{12}=-f_{21} .
$$

Next we want to complete this set of four vector fields $f_{i j}, i, j=1,2$, to a strongly adapted complex frame field. In order to do this, we consider the horizontal leaf $\left.\mathcal{S}\right|_{\mathcal{U}} \times\left\{I_{2}\right\} \subset \mathcal{H}(M)^{\mathrm{qk}}$ and a field of adapted complex frames $\left(e_{ \pm a}\right)$ for $T^{\mathbb{C}} \mathcal{S} \simeq T^{\mathbb{C}}\left(\mathcal{S} \times\left\{I_{2}\right\}\right)$, in which the elements $e_{ \pm a}$ with $a=1,2$ are given by precisely the fields $f_{i a}$,

$$
e_{+1}=f_{11}, \quad e_{+2}=f_{12}, \quad e_{-1}=f_{21}, \quad e_{-2}=f_{22} .
$$

Then, over any other horizontal leaf, $\left.\mathcal{S}\right|_{\mathcal{U}} \times\left\{\left(u_{ \pm}^{i}\right)\right\} \subset \mathcal{H}(M)^{\mathrm{qk}}$, the adapted complex frame field $\left(e_{ \pm a}\right)$ is given by $e_{ \pm a}=u_{ \pm}^{j} e_{i a}$. In particular, by construction,

$$
e_{+1}=u_{+}^{i} f_{i 1}, \quad e_{+2}=u_{+}^{i} f_{i 2}, \quad e_{-1}=u_{-}^{i} f_{i 1}, \quad e_{-2}=u_{-}^{i} f_{i 2} .
$$

\subsection{Prepotentials and instantons}

\subsubsection{The components of the potential of a lifted instanton}

Let $\left(E^{\prime}, D^{\prime}\right)$ be the lift to $\mathcal{H}(M)^{\mathrm{qk}}$ of an instanton $(E, D)$ on a qk manifold $(M, g, Q)$. Consider an open subset $\mathcal{W}$ of $\mathcal{H}(M)^{\mathrm{qk}}$ with holomorphically adapted coordinates $\left(z^{ \pm a}, u_{ \pm}^{i}\right)$ and gauges (trivialisations) for the lifted $G$-bundle $P^{\prime}$ and the associated vector bundle $E^{\prime}$,

$$
\varphi:\left.P^{\prime}\right|_{\mathcal{U}} \rightarrow \mathcal{W} \times G, \quad \hat{\varphi}:\left.E^{\prime}\right|_{\mathcal{U}} \rightarrow \mathcal{W} \times V .
$$

By Corollary 5.2, $\left(E^{\prime}, D^{\prime}\right)$ is the lift of an instanton $\left(E^{\mathcal{S}}, D^{\mathcal{S}}\right)$ on the hk manifold $\mathcal{S}$. Then, by[13, Prop. 4.1], the bundles $P^{\prime}$ and $E^{\prime}$ are real analytic, they have natural structures of holomorphic bundles over $\mathcal{H}(M)^{\mathrm{qk}}$ and the connection 1 -form $\omega^{\prime}$ (corresponding to $D^{\prime}$ ) is invariant under the complex structure of $P^{\prime}$. We may therefore assume that the gauge $\varphi$ is holomorphic and that in this gauge $\omega^{\prime}$ is determined by its $(1,0)$-potential $A^{10}$, the restriction of the potential $A$ to the holomorphic tangent space $T^{10} \mathcal{H}(M)^{\mathrm{qk}}$ of $\mathcal{H}(M)^{\mathrm{qk}}$. Because of real analyticity, we may also assume that the components of $A$ admit unique holomorphic extensions from $\mathcal{W}$ to a neighbourhood $\widetilde{\mathcal{W}}$ of complexified harmonic space $\mathcal{H}^{\mathbb{C}}(M)^{\mathrm{qk}}=\mathcal{H}(M)^{\mathrm{qk}} \times \mathcal{H}(M)^{\mathrm{qk}}$.

We now recall that at each fixed $\left(z_{o}^{ \pm a}, u_{o \pm}^{i}\right) \in \widetilde{\mathcal{W}}$, the vectors

$$
\left.H_{0}\right|_{y_{o}},\left.\quad H_{ \pm \pm}\right|_{y_{o}},\left.\quad \frac{\partial}{\partial z^{ \pm a}}\right|_{z_{o}^{ \pm a}}
$$


constitute a basis for the holomorphic tangent space $T_{y_{o}}^{10} \mathcal{H}^{\mathbb{C}}(M)^{\mathrm{qk}}$. This means that the $(1,0)$-potential $A^{10}$ of $\left(E^{\prime}, D^{\prime}\right)$ is uniquely determined by the $\mathfrak{g}$-valued holomorphic functions

$$
A_{0}:=A\left(H_{0}\right), \quad A_{ \pm \pm}:=A\left(H_{ \pm \pm}\right), \quad A_{ \pm a}:=A\left(\frac{\partial}{\partial z^{ \pm a}}\right) .
$$

In the following, $A_{0}, A_{ \pm \pm}, A_{ \pm a}$ will always denote these $\mathfrak{g}$-valued functions.

\subsubsection{The analytic gauge and the prepotential of an instanton}

Keeping to the notation of the previous subsection, pick a point $\left(z_{o}^{ \pm a}, U_{o}\right) \in \mathcal{W}$ with $U_{o}=I_{2}$ and write $\left.x_{o}=\pi^{\mathrm{qk}}\left(z_{o}^{ \pm a}, U_{o}\right)\right) \in M$. The following theorem follows from [13, Thm. 4.3 and Lemma 4.6] on minor modification of their proofs.

Theorem 5.3 The neighbourhood $\widetilde{\mathcal{W}} \subset \mathcal{H}^{\mathbb{C}}(M)^{\mathrm{qk}}$ and the holomorphic gauge $\varphi:\left.P^{\prime}\right|_{\widetilde{\mathcal{W}}} \rightarrow \widetilde{\mathcal{W}} \times G$ can be chosen in such a way that the components of the instanton potential $A^{10}$ satisfy the conditions

$$
A_{0}=A_{-a}=A_{+1}=A_{+2}=0 .
$$

Moreover,

(1) the component $A_{--}$satisfies the differential conditions

$$
H_{0} \cdot A_{--}=-2 A_{--}, \quad \frac{\partial A_{--}}{\partial z^{-a}}=\frac{\partial A_{--}}{\partial z^{+1}}=\frac{\partial A_{--}}{\partial z^{+2}}=0
$$

(2) the components $A_{++}, A_{+a}$ are uniquely determined by $A_{--}$, being the unique solutions of the differential problem

$$
\begin{aligned}
& H_{0} \cdot A_{++}=2 A_{++}, \quad H_{--} \cdot A_{++}-H_{++} \cdot A_{--}-\left[A_{++}, A_{--}\right]=0, \\
& A_{+a}=-\frac{\partial A_{++}}{\partial z^{-a}} .
\end{aligned}
$$

Proof The proof of the existence of a holomorphic gauge $\varphi:\left.P^{\prime}\right|_{\widetilde{\mathcal{W}}} \rightarrow \widetilde{\mathcal{W}} \times G$ in which $A^{10}$ satisfies (5.14) involves a slight modification to the proof of [13, Thm. 4.3]. Consider the $G$-invariant distributions $\mathcal{D}_{-}^{h}$, generated by the horizontal lifts of the vector fields $\frac{\partial}{\partial z^{-a}}$, and $\mathcal{D}_{-}^{h} \oplus\left\langle H_{0}^{h},\left(\frac{\partial}{\partial z^{+1}}\right)^{h},\left(\frac{\partial}{\partial z^{+2}}\right)^{h}\right\rangle$, where the second summand is generated by the horizontal lifts of $H_{0}, \frac{\partial}{\partial z^{+1}}$ and $\frac{\partial}{\partial z^{+2}}$. Both these distributions are involutive, the second because

(a) $P^{\prime}$, being the lift of an instanton on $M$, has identically vanishing curvature along the infinitesimal transformations of the structure group $L$ of $\mathcal{H}(M)^{\mathrm{qk}}$ over $M$ (see, e.g. [13, Prop. 2.2]), and

(b) both $\frac{\partial}{\partial z^{+1}}$ and $\frac{\partial}{\partial z^{+2}}$ are pointwise linear combinations of the infinitesimal transformations $H_{\alpha}$ and $G_{\beta}$. 
We may now follow the argumentation in the proofs of [13, Thm 4.3 and Lemma 4.6] to obtain all the claimed results except for $\frac{\partial A_{--}}{\partial z^{+j}}=0, j=1,2$. These equations, however, follow from other conditions in (5.14) and (5.16):

$$
\begin{aligned}
0 & =\frac{\partial}{\partial z^{-j}} \cdot\left(H_{--} \cdot A_{++}-H_{++} \cdot A_{--}-\left[A_{++}, A_{--}\right]\right) \\
& =H_{--} \cdot\left(\frac{\partial A_{++}}{\partial z^{-j}}\right)+\frac{\partial A_{--}}{\partial z^{+j}}-\left[\frac{\partial A_{++}}{\partial z^{-j}}, A_{--}\right] \\
& =-H_{--} \cdot A_{+j}+\frac{\partial A_{--}}{\partial z^{+j}}+\left[A_{+j}, A_{--}\right]=\frac{\partial A_{--}}{\partial z^{+j}} .
\end{aligned}
$$

A holomorphic gauge $\varphi: P^{\prime} \rightarrow \widetilde{\mathcal{W}} \times G$ in which the potential $A^{10}$ satisfies (5.14) is called an analytic gauge. The component $A_{--}$of $A^{10}$ in an analytic gauge is the prepotential of the instanton in that gauge .

The above theorem essentially says that an instanton $(E, D)$ on a qk manifold is completely determined its prepotential $A_{--}$in an analytic gauge for the lifted gauge field $\left(E^{\prime}, D^{\prime}\right)$. For hk manifolds this property admits a converse [13, Thm. 5.1]. We now give the corresponding property for qk manifolds.

Theorem 5.4 Let $\mathcal{W} \subset \mathcal{H}(M)^{\mathrm{qk}}$ be an open subset of the harmonic space of a $q \mathrm{k}$ manifold $(M, g, Q)$ on which we may choose holomorphically adapted coordinates $\left(z^{ \pm a}, u_{ \pm}^{i}\right), a=1, \ldots, 2 n+2, i=1,2$ and a trivialisation of the lifted bundle $P^{\prime}$ over $\mathcal{H}(M)^{\mathrm{qk}}$. Given a map $\mathbb{A}_{--}: \mathcal{W} \subset \mathcal{H}(M)^{\mathrm{qk}} \rightarrow \mathfrak{g}$ satisfying

$$
H_{0} \cdot \mathbb{A}_{--}=-2 \mathbb{A}_{--}, \quad \frac{\partial \mathbb{A}_{--}}{\partial z^{-a}}=0, \quad \frac{\partial \mathbb{A}_{--}}{\partial z^{+1}}=\frac{\partial \mathbb{A}_{--}}{\partial z^{+2}}=0
$$

there exists a unique (up to equivalence) instanton $(E, D)$ on the $q k$ manifold $\left(\mathcal{U}:=\pi^{\mathrm{qk}}(\mathcal{W}) \subset M,\left.g\right|_{\mathcal{U}},\left.Q\right|_{\mathcal{U}}\right)$, admitting an analytic gauge in which $\mathbb{A}_{--}$is the prepotential.

Proof By [13, Thm. 5.1] and the first two equations in (5.17), we have existence of an instanton $(\widehat{E}, \widehat{D})$ on the open subset $\mathcal{U}^{\mathcal{S}}=\pi^{\mathcal{H} \mid \mathcal{S}}(\widetilde{\mathcal{W}})$ of the hyperkähler cone $\left(\mathcal{S}, h, \mathbb{J}_{\alpha}\right)$ with prepotential $A_{--}=\mathbb{A}_{--}$. The claim is then proven if $(\widehat{E}, \widehat{D})$ is shown to be the lift of a gauge field on $\mathcal{U}=\pi^{\mathrm{qk}}(\mathcal{W})$ of $M$. By [13, Prop. 2.2], this is the case if and only if two conditions are satisfied: (a) the horizontal lifts to $\widehat{P}$ of the infinitesimal transformations $G_{\alpha}^{\mathcal{S}}$, $\alpha \in\{D, 0,1,2\}$, of the structure group $\mathbb{Q}^{*} / \mathbb{Z}_{2}=L / \mathrm{SL}_{2}(\mathbb{C})$ of the Swann bundle over $M$ are complete vector fields and (b) the curvature $F$ of $(\widehat{E}, \widehat{D})$ vanishes identically when evaluated along any $G_{\alpha}^{\mathcal{S}}$. Condition (a) is surely satisfied: For any trivialisation $\left.P^{\prime}\right|_{\mathcal{U}} \simeq \mathcal{U} \times G$, the $\mathcal{U}$ -component of the horizontal lift of the vector field $G_{\alpha}^{\mathcal{S}}$ is $G_{\alpha}^{\mathcal{S}}$ itself and the $G$-part is the image of $G_{\alpha}^{\mathcal{S}}$ in $\mathfrak{g}$ by applying to it the potential of the connection in this trivialisation. Both components are therefore bounded at all points and the horizontal lifts of the $G_{\alpha}^{\mathcal{S}}$ are therefore complete. It remains to check that (b) holds. Since the vector fields $G_{\alpha}^{\mathcal{S}}$ are pointwise linear combinations of the complex vector fields $\frac{\partial}{\partial z^{ \pm j}}, j=1,2$, we only need to check that 


$$
F\left(\frac{\partial}{\partial z^{+j}}, \frac{\partial}{\partial z^{-a}}\right)=F\left(\frac{\partial}{\partial z^{-j}}, \frac{\partial}{\partial z^{+a}}\right)=0, \quad j=1,2, a=1, \ldots, 2 n+2 .
$$

By [13, (5.19)], this holds if and only if

$$
\frac{\partial^{2} A_{++}}{\partial z^{-j} \partial z^{-a}}=0
$$

Now, recall (see $[13,(5.20)])$ that the component $A_{++}$of the lift of $(\widehat{E}, \widehat{D})$ to $\mathcal{W}$ satisfies the differential equation

$$
H_{--} \cdot A_{++}-H_{++} \cdot \mathbb{A}_{--}+\left[\mathbb{A}_{--}, A_{++}\right]=0 .
$$

Differentiating this equation along $\frac{\partial}{\partial z^{-j}}, j=1,2$, and using (5.17), we obtain

$$
H_{--} \cdot \frac{\partial A_{++}}{\partial z^{-j}}+\left[\mathbb{A}_{--} \frac{\partial A_{++}}{\partial z^{-j}}\right]=0 .
$$

Thus, the $G$-equivariant extensions of the functions $g_{+j}:=\frac{\partial A_{++}}{\partial z^{-j}}$ on $P^{\prime}$ satisfy the differential problem

$$
H_{0}^{h} \cdot g_{+j}=g_{+j}, \quad H_{--}^{h} \cdot g_{+j}=0,
$$

with $H_{0}^{h}$ and $H_{--}^{h}$ being horizontal lifts to $P^{\prime}$ of $H_{0}$ and $H_{--}$, respectively. Now, using [12, Lemma 5.3], in the version obtained by replacing $H_{++}$by $H_{--}$, shows that this system only has a trivial solution, $\frac{\partial A_{++}}{\partial z^{-i}}=\left.g_{+i}\right|_{\mathcal{H}(M)^{\mathrm{qk}} \times\{e\}}=0$, which yields the required condition (5.18).

\subsubsection{Equivalent prepotentials and curvatures}

Let $\mathcal{W} \subset \mathcal{H}(M)^{\mathrm{qk}}$ be an open subset of the harmonic space of $(M, g, Q)$ admitting a set of holomorphically adapted coordinates $\left(z^{ \pm a}, u_{ \pm}^{i}\right)$ and a trivialisation of the lifted bundle $P^{\prime}$ associated with a vector bundle $E$.

As shown by Theorems 5.3 and 5.4, instantons $(E, D)$ are completely determined by their prepotentials, the real analytic maps $A_{--}: \mathcal{W} \rightarrow \mathfrak{g}$ satisfying (5.17). Two distinct $\mathfrak{g}$-valued prepotentials $A_{--}, A_{--}^{\prime}$ on $\mathcal{W}$ correspond to two equivalent instantons if and only if they are the $H_{--}$-components of two $(1,0)$-potentials $A^{10}, A^{\prime 10}$, both in an analytic gauge (i.e. satisfying (5.14)) and related to each other by a gauge transformation (2.2) with $g: \mathcal{W} \rightarrow \mathfrak{g}$.

On the other hand, if the exponential map of the structure group $G$ is surjective (as, e.g. when $G$ is reductive), any gauge transformation $g: \mathcal{W} \rightarrow G$ can be written in the form

$$
g\left(z^{i}, u_{ \pm}^{j}\right)=e^{\widehat{g}\left(z^{i}, u_{ \pm}^{j}\right)} \quad \text { for some appropriate } \widehat{g}: \mathcal{U} \rightarrow \mathfrak{g} .
$$

In this case, $A_{--}, A_{--}^{\prime}$ correspond to equivalent instantons if and only if

$$
A_{--}^{\prime}=\operatorname{Ad}_{e^{-\hat{g}}}\left(A_{--}+H_{--} \cdot \hat{g}\right)
$$

for some $\widehat{g}: \mathcal{U} \rightarrow \mathfrak{g}$ such that $\quad H_{0} \cdot \widehat{g}=\frac{\partial \widehat{g}}{\partial z^{-a}}=\frac{\partial \widehat{g}}{\partial z^{+1}}=\frac{\partial \widehat{g}}{\partial z^{+2}}=0$.

In particular, $A_{--}$corresponds to a flat connection if and only if $A_{--}=-H_{--} \cdot \hat{g}$ for some $\mathfrak{g}$ -valued map $\hat{g}: \mathcal{W} \rightarrow \mathfrak{g}$ satisfying (5.20). 
We now recall that the curvature $\mathcal{F}^{\varphi}$ of the $(1,0)$ potential $A^{10}$ of an instanton on $\mathcal{W} \subset \mathcal{H}(M)^{\mathrm{qk}}$, defined in (2.4), is determined by the $A_{++}=A^{10}\left(H_{++}\right)$component by the simple formula [13, (5.19)],

$$
\mathcal{F}^{\varphi}\left(\frac{\partial}{\partial z^{+a}}, \frac{\partial}{\partial z^{-b}}\right)=\frac{\partial^{2} A_{++}}{\partial z^{+a} \partial z^{+b}} .
$$

Due to this simple relation with the curvature, the component $A_{++}$may be thought of as a second prepotential of the instanton $(E, D)$ on $M$ [13]. By Theorem 5.3, the potential $A^{10}$, and thus the component $A_{++}$, is uniquely determined by the prepotential $A_{--}$. A direct determination of $A_{++}$from $A_{--}$follows from the following (cf. [13, Prop. 5.5]):

Proposition 5.5 Given a prepotential $A_{--}: \mathcal{W} \subset \mathcal{H}(M)^{\mathrm{qk}} \rightarrow \mathfrak{g}$ for a lifted instanton in an analytic gauge, the corresponding second prepotential $A_{++}$coincides with the unique solution on $\mathcal{W}$ of the differential problem for the unknown $B_{++}$,

$$
H_{--} \cdot B_{++}=H_{++} \cdot A_{--}-\left[A_{--}, B_{++}\right], \quad H_{0} \cdot B_{++}=2 B_{++} \cdot
$$

The horizontal projection of $B_{++}$in the bundle $\mathcal{H}(M)^{\mathrm{qk}} \rightarrow M$ is known as the Leznov field and plays a rôle in various contexts (see, e.g. [8-11, 19, 28]).

\section{Prepotentials in canonical forms}

\subsection{Potentials in canonical form for gauge fields of compact type}

\subsubsection{The canonical connection of a Stiefel bundle}

Let $\left(e_{1}^{o}, \ldots, e_{m+k}^{o}\right)$ be the standard basis of $\mathbb{C}^{m+k}$ and denote by $\mathcal{V}_{m+k, m}$ the Stiefel manifold of unitary $m$-frames in $\mathbb{C}^{m+k}$, i.e. the manifold of all ordered $m$-tuples $\left(v_{1}, \ldots, v_{m}\right)$ of complex vectors $v_{i}=b_{i}^{\alpha} e_{\alpha}^{o}$ given by some $B:=\left(b_{i}^{\alpha}\right) \in \mathbb{C}_{(m+k) \times m}$ such that

$$
B^{\dagger} B=\mathrm{I}_{m}, \quad \text { where } B^{\dagger}:=\overline{B^{T}} .
$$

Note that for any such unitary $m$-frame, the associated linear map

$$
P_{B}: \mathbb{C}^{m+k} \rightarrow \mathbb{C}^{m+k}, \quad P_{B}\left(\begin{array}{c}
x^{1} \\
\vdots \\
x^{m+k}
\end{array}\right):=B B^{\dagger}\left(\begin{array}{c}
x^{1} \\
\vdots \\
x^{m+k}
\end{array}\right)
$$

is the unitary projector from $\mathbb{C}^{m+k}$ onto the $m$-plane spanned by the $v_{i}$. In particular, $P_{B}^{2}=P_{B}$. The manifold $\mathcal{V}_{m+k, m}$ is identifiable with the homogeneous space $\mathcal{V}_{m+k, m}=\mathrm{U}_{m+k} /\left(\left\{\mathrm{I}_{m}\right\} \times \mathrm{U}_{k}\right)$ and its natural projection onto the Grassmannian of $m$-planes

$$
\pi: \mathcal{V}_{m+k, m} \rightarrow \mathrm{Gr}_{m}\left(\mathbb{C}^{m+k}\right) \simeq \mathrm{U}_{m+k} /\left(\mathrm{U}_{m} \times \mathrm{U}_{k}\right)
$$

makes it a principal $\mathrm{U}_{k}$-bundle over $\mathrm{Gr}_{m}\left(\mathbb{C}^{m+k}\right)$. Consider the map $\mathcal{M}: \mathcal{V}_{m+k, k} \rightarrow \mathbb{C}_{(m+k) \times k}$ which sends each $m$-frame

$$
\left(v_{1}:=B_{1}^{\alpha} e_{\alpha}^{o}, \ldots, v_{m}:=B_{m}^{\alpha} e_{\alpha}^{o}\right)
$$


into the corresponding matrix $B=\left(B_{i}^{\alpha}\right)$. The $\mathbb{C}_{m \times m}$-valued 1-form on $\mathcal{V}_{m+k, m}$

$$
\omega^{\text {can }}=\mathcal{M}^{\dagger} d \mathcal{M}
$$

is then $\mathfrak{t}_{m}$-valued. Further, it can be checked that it is a connection 1 -form on the $\mathrm{U}_{m}$-bundle (6.3), called the canonical connection of $\mathcal{V}_{m+k, m}[21]$.

\subsubsection{Potentials in canonical form}

Let $G^{o}$ be a compact Lie group, which, with no loss of generality, we assume to be a closed subgroup of a unitary group $\mathrm{U}_{m}$ for some integer $m$. The following crucial fact has been proved by Narasimhan and Ramanan.

Theorem 6.1 ([21]) Let $\left(E^{o}, D^{o}\right)$ be a gauge field of compact type over an n-dimensional manifold $M$, associated with a principal bundle with connection $\left(P^{o}, \omega^{o}\right)$ having structure group $G^{o} \subset \mathrm{U}_{m}$. Then, there exists a $G^{o}$ equivariant embedding

$$
\Phi: P^{o} \rightarrow \mathcal{V}_{m+k, m} \quad \text { with } \quad k=(n+1)(2 n+1) m^{2}-m,
$$

such that $\omega^{o}=\Phi^{*}\left(\omega^{\mathrm{can}}\right)=\mathcal{M}^{\Phi \dagger} d \mathcal{M}^{\Phi}$ with $\mathcal{M}^{\Phi}=\mathcal{M} \circ \Phi$.

This implies that, in a fixed trivialisation $\left.P^{o}\right|_{\mathcal{U}} \simeq \mathcal{U} \times G^{o}$, the potential $A$ of the connection 1-form $\omega^{o}$ can always be written as

$$
A=\xi^{\dagger} d \xi, \quad \text { where } \quad \xi:=\left.\left(\mathcal{M}^{\Phi}\right)\right|_{\mathcal{U} \times\{e\}}=: \mathcal{U} \rightarrow \mathbb{C}_{(m+k) \times m} .
$$

Note that by construction, $\xi_{x}^{\dagger} \xi_{x}=\mathrm{I}_{m}$ at any $x \in \mathcal{U}$. Observe also that:

(1) If $A^{\prime}$ is the potential of $\omega^{o}$ in a new gauge, related to $A$ by a gauge transformation determined by a map $g: \mathcal{U} \rightarrow G^{o} \subset \mathrm{U}_{m}$, then $A^{\prime}$ also has the form (6.6), with $\xi$ replaced by $\xi^{\prime}=\xi g$. This is due to the fact that

$$
A^{\prime}=\operatorname{Ad}_{g^{-1}} A+g^{-1} d g=\left(g^{\dagger} \xi^{\dagger}\right)(d \xi g)+\left(g^{\dagger} \xi^{\dagger}\right)(\xi d g)=(\xi g)^{\dagger} d(\xi g) .
$$

(2) From the definition of the map $\xi=\left.\mathcal{M}^{\Phi}\right|_{\mathcal{U} \times\{e\}}$, after possibly restricting the open set $\mathcal{U}$, it is always possible to determine a row-permuting matrix $C \in \mathrm{U}_{m+k}$ such that the upper $m \times m$ block $h_{x}$ of the product $C \xi_{x} \in \mathbb{C}_{(m+k) \times m}$ is of maximal rank for all $x \in \mathcal{U}$. This determines a map $h: \mathcal{U} \rightarrow \mathrm{GL}_{m}(\mathbb{C})$ such that $C \xi h^{-1}$ has the form

$$
C \xi h^{-1}=\left(\begin{array}{c}
\mathrm{I}_{m} \\
\lambda
\end{array}\right) \quad \text { for some map } \lambda: \mathcal{U} \rightarrow \mathbb{C}_{k \times m}
$$

uniquely determined by $\xi$ and the constant matrix $C$.

(3) Conversely, given a smooth map $\lambda: \mathcal{U} \rightarrow \mathbb{C}_{k \times m}$ we can find a row-permuting matrix $C$ and a map $\xi: \mathcal{U} \rightarrow \mathbb{C}_{(m+k) \times m}$ which determine $\lambda$. For example, $C=\mathrm{I}_{m+k}$ and

$$
\xi=\xi^{(\lambda)}:=\left(\begin{array}{c}
\mathrm{I}_{m} \\
\lambda
\end{array}\right)\left(\mathrm{I}_{m}+\lambda^{\dagger} \lambda\right)^{-\frac{1}{2}}
$$

will do.

(4) Consider two smooth maps $\lambda, \lambda^{\prime}: \mathcal{U} \rightarrow \mathbb{C}_{k \times m}$ and the $\mathrm{U}_{k}$-valued maps 


$$
A^{(\lambda)}=\xi^{(\lambda) \dagger} d \xi^{(\lambda)}, \quad A^{\left(\lambda^{\prime}\right)}=\xi^{\left(\lambda^{\prime}\right) \dagger} d \xi^{\left(\lambda^{\prime}\right)}
$$

with $\xi^{(\lambda)}, \xi^{\left(\lambda^{\prime}\right)}$ as in (6.9). The maps $A^{(\lambda)}$ and $A^{\left(\lambda^{\prime}\right)}$ are two potentials of the same connection in two distinct gauges if and only if there exist two row-permuting matrices $C, C^{\prime} \in \mathrm{U}_{m+k}$ and a map $g: \mathcal{U} \rightarrow G^{o} \subset \mathrm{U}_{m}$, such that $C^{\prime} \xi^{\left(\lambda^{\prime}\right)}=C \xi^{(\lambda)} g$. On the other hand, since $C^{\dagger}=C^{-1}$ and $C^{\prime \dagger}=C^{\prime-1}$, we see that $A^{(\lambda)}$ and $A^{\left(\lambda^{\prime}\right)}$ are independent of $C$ and $C^{\prime}$ and, consequently, that there is no loss of generality in assuming that $C=C^{\prime}=\mathrm{I}_{m+k}$. Hence, $A^{(\lambda)}$ and $A^{\left(\lambda^{\prime}\right)}$ are gauge equivalent if and only if

$$
\left(\begin{array}{c}
\mathrm{I}_{m} \\
\lambda
\end{array}\right)=\left(\begin{array}{c}
\mathrm{I}_{m} \\
\lambda^{\prime}
\end{array}\right) \widetilde{g} \quad \text { with } \quad \tilde{g}=\left(\mathrm{I}_{m}+\lambda^{\dagger} \lambda\right)^{\frac{1}{2}}\left(\mathrm{I}_{m}+\lambda^{\prime \dagger} \lambda^{\prime}\right)^{-\frac{1}{2}} g .
$$

This holds if and only if $\widetilde{g}=\mathrm{I}_{m}$ and $\lambda=\lambda^{\prime}$, from which $g=\mathrm{I}_{m}$ follows.

These observations imply that in each gauge equivalence class of potentials, there exists exactly one potential of the form $A^{(\lambda)}=\xi^{(\lambda) \dagger} d \xi^{(\lambda)}$ for some map $\lambda: \mathcal{U} \rightarrow \mathbb{C}_{(m+k) \times m}$. The number of distinct $\lambda$ 's which determine the same potential $A^{(\lambda)}$ is less than or equal to $(m+k)$ !, the cardinality of the group of row-permuting matrices $C$.

We have therefore verified the following direct consequence of Theorem 6.1.

Corollary 6.2 For any gauge field $\left(E^{o}, D^{o}\right)$ with a structure group $G^{o} \subset \mathrm{U}_{m}$, there exists an integer $k \geq 0$ with the property that, around any $x_{o} \in M$, there is a neighbourhood $\mathcal{U}$ and a gauge $\varphi:\left.P^{o}\right|_{\mathcal{U}} \rightarrow \mathcal{U} \times G$, in which the potential $A: T^{*} \mathcal{U} \rightarrow \mathfrak{g}^{o}$ has the form

$$
A=A^{(\lambda)}=\xi^{(\lambda) \dagger} d \xi^{(\lambda)}
$$

with $\xi^{(\lambda)}$ as in (6.9) for some smooth $\lambda: \mathcal{U} \rightarrow \mathbb{C}_{k \times m}$. Such a gauge is uniquely determined by the restricted gauge field $\left.\left(E^{o}, D^{o}\right)\right|_{\mathcal{U}}$ and the map $\lambda$ is uniquely determined up to the action of a row-permuting matrix $C$, according to Eqs. (6.8) and (6.9).

The potential (6.10) is said to be in canonical form and we call the corresponding map $\lambda: \mathcal{U} \rightarrow \mathbb{C}_{k \times m}$ its core.

\subsection{The expression of the curvature in terms of the core}

Let $A^{(\lambda)}=\xi^{(\lambda) \dagger} d \xi^{(\lambda)}: \mathcal{U} \rightarrow \mathfrak{g}^{o}$ be a potential in canonical form with core $\lambda$ for a gauge field $\left(P^{o}, \omega^{o}\right)$ with structure group $G^{o}$. For such potential, we now select a smooth map $\eta: \mathcal{U} \rightarrow \mathbb{C}_{(m+k) \times k}$ with the following property: for each $x \in \mathcal{U}$ the set of columns of the matrix $\eta_{x}$ complete the set of columns of $\xi_{x}^{(\lambda)}$ to a unitary basis for the vector space $\mathbb{C}_{(m+k) \times(m+k)}$. In other words, we assume that $\eta$ is such that

$$
\text { the matrix }\left(\xi_{x}^{(\lambda)} \mid \eta_{x}\right) \text { is in } \mathrm{U}_{m+k} \quad \text { for each } x \in \mathcal{U} \text {. }
$$

From the above characterisation, we clearly have that $\eta^{\dagger} \eta=\mathrm{I}_{k}$. Moreover, since the columns of $\eta_{x}$ are linearly independent from those of $\xi_{x}$, we may always assume that $\eta$ has the form

$$
\eta=\left(\begin{array}{c}
v \\
\mathrm{I}_{k}
\end{array}\right)\left(\mathrm{I}_{k}+v^{\dagger} v\right)^{-\frac{1}{2}} \quad \text { where } v: \mathcal{U} \rightarrow \mathbb{C}_{m \times k}
$$


Note that the columns of such a map $\eta$ are orthogonal to all the columns of $\xi^{(\lambda)}$ if and only if

$$
v^{\dagger}+\lambda=0
$$

a condition which uniquely determines $\eta$ in terms of $\lambda$. In what follows, we denote the map (6.12) by $\eta^{(\nu)}$ and we say that $v=-\lambda^{\dagger}$ is the conjugate core of the potential $A=A^{(\lambda)}$.

Clearly a core $\lambda$ and the associated map $\xi^{(\lambda)}$ are uniquely determined by the conjugate core $v=-\lambda^{\dagger}$ and the corresponding map $\eta^{(\nu)}$, and vice versa.

The following lemma (see, e.g. [3, Ch. II.3]) gives a simple and convenient expression for the curvature of a connection in terms of the two maps $\xi^{(\lambda)}$ and $\eta^{(\nu)}$.

Lemma 6.3 Let $A^{(\lambda)}=\xi^{(\lambda) \dagger} d \xi^{(\lambda)}: \mathcal{U} \rightarrow \mathfrak{g}^{o}$ be a potential in canonical form with core $\lambda$ and $v=-\lambda^{\dagger}$. The curvature $F$ of the corresponding gauge field is

$$
F=\xi^{(\lambda) \dagger}\left\{\left(\begin{array}{cc}
d v \wedge\left(\mathrm{I}_{2 k}+v^{\dagger} v\right)^{-1} d v^{\dagger} & 0 \\
0 & 0
\end{array}\right)\right\} \xi^{(\lambda)} .
$$

Proof Since $\xi^{(\lambda) \dagger} \xi^{(\lambda)}=\mathrm{I}_{m}$ and $\xi^{(\lambda) \dagger} d \xi^{(\lambda)}=-d \xi^{(\lambda) \dagger} \xi^{(\lambda)}$,

$$
\begin{aligned}
\xi^{(\lambda) \dagger}\left\{d\left(\xi^{(\lambda)} \xi^{(\lambda) \dagger}\right) \wedge d\left(\xi^{(\lambda)} \xi^{(\lambda) \dagger}\right)\right\} \xi^{(\lambda)} & =d \xi^{(\lambda) \dagger} \wedge d \xi^{(\lambda)}+\xi^{(\lambda) \dagger} d \xi^{(\lambda)} \wedge \xi^{(\lambda) \dagger} d \xi^{(\lambda)} \\
& =d A^{(\lambda)}+A^{(\lambda)} \wedge A^{(\lambda)}=F .
\end{aligned}
$$

On the other hand, since $\left(\xi_{x}^{(\lambda)} \mid \eta_{x}^{(\nu)}\right) \in \mathrm{U}_{m+k}$ for each $x \in \mathcal{U}$, we have

$$
\begin{aligned}
& \xi^{(\lambda) \dagger} \eta^{(\nu)}=\xi^{(\lambda) \dagger}\left(\begin{array}{c}
v \\
\mathrm{I}_{2 k}
\end{array}\right)\left(\mathrm{I}_{2 k}+v^{\dagger} v\right)^{-\frac{1}{2}}=0 \\
& \Longrightarrow \xi^{(\lambda) \dagger}\left(\begin{array}{c}
v \\
\mathrm{I}_{k}
\end{array}\right)=0 \quad \text { and } \mathrm{I}_{k+m}=\xi^{(\lambda)} \xi^{(\lambda) \dagger}+\eta^{(\nu)} \eta^{(\nu) \dagger}
\end{aligned}
$$

This implies that

$$
\begin{aligned}
\xi^{(\lambda) \dagger} & \left\{d\left(\xi^{(\lambda)} \xi^{(\lambda) \dagger}\right) \wedge\left(\xi^{(\lambda)} \xi^{(\lambda) \dagger}\right)\right\} \xi^{(\lambda)} \\
& =\xi^{(\lambda) \dagger}\left\{d\left(\mathrm{I}_{m+k}-\eta^{(\nu)} \eta^{(\nu) \dagger}\right) \wedge d\left(\mathrm{I}_{m+k}-\eta^{(\nu)} \eta^{(\nu) \dagger}\right)\right\} \xi^{(\lambda)} \\
& =\xi^{(\lambda) \dagger}\left(d\left(\eta^{(\nu)} \eta^{\nu) \dagger}\right) \wedge d\left(\eta^{(\nu)} \eta^{(\nu) \dagger}\right)\right) \xi^{(\lambda)} \\
& =\xi^{(\lambda) \dagger}\left(\left(\begin{array}{c}
d \nu \\
0
\end{array}\right) \wedge\left(\mathrm{I}_{2 k}+v^{\dagger} v\right)^{-1}\left(d \nu^{\dagger} 0\right)\right) \xi^{(\lambda)} .
\end{aligned}
$$

From this and (6.15) the conclusion follows.

\subsection{Prepotentials in canonical form}

We now apply the above results to instantons on a qk manifold $(M, g, Q)$ and its harmonic space $\mathcal{H}(M)^{\mathrm{qk}}$. Let $\mathcal{W} \subset \mathcal{H}(M)^{\mathrm{qk}}$ be an open subset of the harmonic space admitting holomorphically adapted coordinates $\left(z^{ \pm a}, u_{ \pm}^{i}\right)$ and $G^{o}$ a compact Lie group with complexification $G$. By compactness, we may always assume that $G^{o} \subset \mathrm{U}_{m}$ and $\mathfrak{g}^{o}=\operatorname{Lie}\left(G^{0}\right) \subset \mathfrak{a}_{m}$ for some $m \geq 1$. 
We denote by $\left(E^{o}, D^{o}\right)$ an instanton on $(M, g, Q)$ with structure group $G^{o}$ and associated principal bundle with connection $\left(P^{o}, \omega^{o}\right)$, and by $\left(E^{\prime o}, D^{\prime o}\right)$ and $\left(P^{o \prime}, \omega^{\prime o}\right)$ the corresponding lifts to $\mathcal{H}(M)^{\mathrm{qk}}$. From Theorem 5.4, Corollary 6.2 and Lemma 6.3, we obtain

Theorem 6.4 Let $\lambda: \mathcal{W} \simeq \mathcal{W} \times\left\{I_{2}\right\} \rightarrow \mathbb{C}_{k \times m}$ be a smooth map, with associated map $\xi^{(\lambda)}$ defined in (6.9) and $v=-\lambda^{\dagger}$, such that for each $x \in \mathcal{W} \times\left\{I_{2}\right\}$ the following hold:

$$
\begin{aligned}
& \left.\xi^{(\lambda) \dagger}\left(\frac{\partial \xi^{(\lambda)}}{\partial z^{+a}}+\frac{\partial \xi^{(\lambda)}}{\partial z^{-a}}\right)\right|_{x} \in \mathfrak{g}^{o}, \\
& \frac{\partial \lambda}{\partial z^{ \pm 1}}=\frac{\partial \lambda}{\partial z^{ \pm 2}}=H_{0} \cdot \lambda=H_{++} \cdot \lambda=H_{--} \cdot \lambda=0, \\
& \left(d \nu \wedge\left(\mathrm{I}_{2 k}+v^{\dagger} \nu\right)^{-1} d \nu^{\dagger}\right)\left(\frac{\partial}{\partial z^{ \pm a}}, \frac{\partial}{\partial z^{ \pm b}}\right)=0, \\
& \left(d v \wedge\left(\mathrm{I}_{2 k}+v^{\dagger} v\right)^{-1} d v^{\dagger}\right)\left(\frac{\partial}{\partial z^{+a}}, \frac{\partial}{\partial z^{-b}}\right) \\
& =-\left(d v \wedge\left(\mathrm{I}_{2 k}+v^{\dagger} v\right)^{-1} d v^{\dagger}\right)\left(\frac{\partial}{\partial z^{-a}}, \frac{\partial}{\partial z^{+b}}\right) .
\end{aligned}
$$

Then:

(1) For each $y \in \mathcal{W}$, there exists a neighbourhood $\mathcal{W}^{\prime} \subset \mathcal{W}$ of $y$ on which there is a solution $\mu^{(\lambda)}: \mathcal{W}^{\prime} \subset \mathcal{W} \rightarrow \mathfrak{g}$ to the system of partial differential equations

$$
H_{0} \cdot \mu=0, \quad \frac{\partial \mu}{\partial z^{-a}}=\xi^{(\lambda) \dagger} \frac{\partial \xi^{(\lambda)}}{\partial z^{-a}}, \quad \frac{\partial \mu}{\partial z^{+1}}=\frac{\partial \mu}{\partial z^{+2}}=0 .
$$

(2) The map

$$
A_{--}^{(\lambda)}: \mathcal{W}^{\prime} \rightarrow \mathfrak{g}, \quad A_{--}^{(\lambda)}:=\operatorname{Ad}_{e^{-\mu}}\left(\xi^{(\lambda) \dagger}\left(H_{--} \cdot \xi^{(\lambda)}\right)+H_{--} \cdot \mu\right)
$$

is the prepotential of an instanton $\left(\left.E^{o}\right|_{\mathcal{U}}, D^{o}\right)$ on $\mathcal{U}:=\pi^{\mathrm{qk}}\left(\mathcal{W}^{\prime}\right) \subset M$ with structure group $G^{o}$ in some analytic gauge.

Further, any prepotential of an instanton on $(M, g, Q)$ with structure group $G^{o}$ is gauge equivalent to one of the form (6.19) for some $\lambda$ satisfying (6.17) and a solution $\mu$ of (6.18). Finally, two prepotentials $A_{--}^{(\lambda)}, A_{--}^{\left(\lambda^{\prime}\right)}$, determined by maps $\lambda, \lambda^{\prime}: \mathcal{W}^{\prime} \rightarrow \mathbb{C}_{k \times m}$, are gauge equivalent if and only if the corresponding 1-forms $A^{o(\lambda)}=\xi^{(\lambda) \dagger} d \xi^{(\lambda)}$ and $A^{o\left(\lambda^{\prime}\right)}=\xi^{\left(\lambda^{\prime}\right) \dagger} d \xi^{\left(\lambda^{\prime}\right)}$ are equal $\left(^{2}\right)$.

Proof Assume that $\lambda$ satisfies (6.17) and set

$$
A^{o(\lambda)}: T^{*} \mathcal{W} \rightarrow \mathbb{C}_{m \times m},\left.\quad A^{o(\lambda)}\right|_{x}:=\xi^{(\lambda) \dagger} d \xi^{(\lambda)} .
$$

By the first condition in (6.17), the map $A^{o(\lambda)}$ is $\mathfrak{g}^{o}$ valued and can be considered as the potential of a connection 1-form $\omega^{o}$ on the trivial bundle $\pi^{\prime}: P^{o}=\mathcal{W} \times G^{o} \rightarrow \mathcal{W}$ and of the corresponding gauge field $\left(E^{\prime}, D^{\prime}\right)$. By Lemma 6.3 and Proposition 5.1, the other conditions in (6.17) imply that $\left(E^{\prime}, D^{\prime}\right)$ is the lift of an instanton on $\mathcal{U}=\pi(\mathcal{W}) \subset M$. By

\footnotetext{
${ }^{2}$ This holds if and only if $\lambda$ coincides with $\lambda^{\prime}$ up to the action of a row-permuting matrix in $\mathrm{GL}_{m+k}(\mathbb{C})$ as mentioned in Corollary 6.2 .
} 
Theorem 5.3 for each $y \in \mathcal{W}$, there exists a neighbourhood $\mathcal{W}^{\prime}$ of $y$ and an analytic gauge for $\left.P^{\prime}\right|_{\mathcal{W}^{\mathcal{W}}}=\left(\left.P^{\prime o}\right|_{\mathcal{W}}\right)^{\mathbb{C}}$, in which the potential satisfies (5.14). This is tantamount to saying that there exists a solution to (6.18) on $\mathcal{W}^{\prime}$, i.e. that (1) holds. Claim (2) follows immediately from the fact that $A^{(\lambda)}=\operatorname{Ad}_{e^{-\mu}}\left(\xi^{(\lambda) \dagger}\left(H_{--} \cdot \xi^{(\lambda)}\right)+d \mu\right)$ is the potential of the lift of an instanton in an analytic gauge.

The property that any instanton $\left(E^{o}, D^{o}\right)$ admits a prepotential of the form (6.19) is checked as follows. By Corollary 6.2, there exists a gauge for the lift $\left(E^{\prime o}, D^{\prime o}\right)$ on $\mathcal{H}(M)^{\mathrm{qk}}$ in which the potential has the form $\widetilde{A}^{(\lambda)}=\xi^{(\lambda) \dagger} d \xi^{(\lambda)}$ for an appropriate choice of a matrixvalued function $\lambda$. The map $\xi^{(\lambda)}$ which gives the potential in this gauge is determined by pulling back the map $\mathcal{M}: \mathcal{V}_{m+k, k} \rightarrow \mathbb{C}_{(m+k) \times k}$ in Sect. 6.1.1 using an appropriate $G^{o}$ equivariant embedding $\Phi^{\prime}: P^{\prime o} \rightarrow \mathcal{V}_{m+k, m}$ into the Stiefel bundle $\mathcal{V}_{m+, k}$. Since $\left(E^{\prime o}, D^{\prime o}\right)$ is the lift of a gauge field on $M$, there is no loss of generality in assuming that this equivariant embedding $\Phi^{\prime}$ has the form $\Phi^{\prime}=\Phi \circ \pi^{\mathrm{qk}}$ for some $G_{o}$-equivariant embedding of $P^{o}$ into $\mathcal{V}_{m+k, m}$. Under this hypothesis, the proof of Corollary 6.2 implies that the function $\lambda$ is constant along the fibres of the bundle $\pi^{\mathrm{qk}}: \mathcal{H}(M)^{\mathrm{qk}} \rightarrow M$ and hence satisfies the first two lines of (6.17). The third line is immediate from (6.14) and the fact that $\widetilde{A}^{(\lambda)}$ satisfies (5.1). By Theorem 5.3, we know that there exists a gauge transformation $g=e^{\mu}: \mathcal{U} \rightarrow G$ which modifies the above gauge of $\left.P^{o}\right|_{\mathcal{W}}$ (and of its complexification $\left.P\right|_{\mathcal{W}}$ ) into an analytic gauge. The new potential given by such a gauge transformation has the component $A_{--}=A\left(H_{--}\right)$ as in (6.19).

For the final claim, we observe that $A_{--}^{(\lambda)}$ and $A_{--}^{\left(\lambda^{\prime}\right)}$ are, respectively, components of potentials which are obtained from the potentials $A^{o(\lambda)}$ and $A^{o\left(\lambda^{\prime}\right)}$ of the $G^{o}$-bundle $P^{o}$ by a gauge transformation in the complexification $P=\left(P^{o}\right)^{\complement}$. Hence $A_{--}^{(\lambda)}$ is gauge equivalent to $A_{--}^{\left(\lambda^{\prime}\right)}$ if and only if $A^{o(\lambda)}$ is gauge equivalent to $A^{o\left(\lambda^{\prime}\right)}$. By Corollary 6.2, this holds if and only if $A^{o(\lambda)}=A^{o\left(\lambda^{\prime}\right)}$.

We say that a prepotential of the form $A_{--}^{(\lambda)}$ is in canonical form and the maps $\lambda$ and $\nu=-\lambda^{\dagger}$ are its core and conjugate core, respectively.

\subsection{Examples of cores for prepotentials in canonical form on $\Vdash P^{n}$}

We now consider the basic example of qk manifold $\mathbb{H} P^{n}=\mathrm{Sp}_{n+1} / \mathrm{Sp}_{n}$, equipped with the $\mathrm{Sp}_{n+1}$-invariant Riemannian metric described in Sect. 3.5. In this concluding section, we present some examples of cores $\lambda$, which determine prepotentials for instantons on (open subsets of) $\mathbb{H} P^{n}$, with structure group $G_{o}=\mathrm{Sp}_{m}$. They correspond to the instantons on $\mathbb{H} P^{n}$ constructed in [20] and are natural generalisations of some of the instantons on $S^{4}=\mathbb{H} P^{1}$ appearing in the ADHM classification [3,4].

From the discussion in Sect. 3.5, the Swann bundle and the harmonic space of $\mathbb{W} P^{n}$ are $\mathbb{M}^{n+1} \backslash\{0\} / \mathbb{Z}_{2}$ and $\left(\left(\mathbb{Q}^{n+1} \backslash\{0\}\right) / \mathbb{Z}_{2}\right) \times \mathrm{SL}_{2}(\mathbb{C})$, respectively. Let us identify $\mathcal{U}_{0}=\left\{\left(q_{0}, \ldots, q_{n}\right) \in \mathbb{\boxplus}^{n+1}: q_{0} \neq 0\right\} \subset \mathbb{\boxplus}^{n+1}$ with a subset of $\mathbb{C}^{2 n+2}$ using the composition of the following two maps: First,

$$
\left(q^{0}, q^{1}, \ldots, q^{n}\right) \in \mathcal{U}_{0} \mapsto\left(q^{0}, r^{1}:=q^{1}\left(q^{0}\right)^{-1}, \ldots, r^{n}:=q^{n}\left(q^{0}\right)^{-1}\right) \in \mathbb{H}^{*} \times \mathbb{H}^{n},
$$

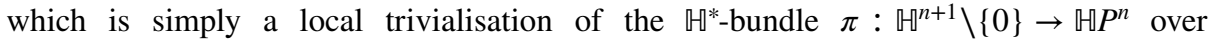
$\mathcal{U}_{0} \subset \mathbb{H} P^{n}$, and second 


$$
\begin{aligned}
\mathbb{H}^{*} & \times \mathbb{H}^{n} \ni\left(q^{0}=\zeta^{0}+\zeta^{0^{\prime}} \mathbf{j} ; r^{1}=\zeta^{1}+\zeta^{1^{\prime}} \mathbf{j}, \ldots, r^{n}=\zeta^{n}+\zeta^{n^{\prime}} \mathbf{j}\right) \\
& \longmapsto\left(\zeta^{0}, \zeta^{0^{\prime}}, \zeta^{1}, \zeta^{1^{\prime}}, \ldots, \zeta^{n}, \zeta^{n^{\prime}}\right) \in \mathbb{C}^{2 n+2} .
\end{aligned}
$$

This identification allows any point of $\mathcal{U}_{0}$ to be considered as a point of the complexification $\left(\mathbb{C}^{2 n+2}\right)^{\mathbb{C}} \simeq \mathbb{C}^{2} \otimes \mathbb{C}^{2 n+2}$ using the embedding

$$
\begin{aligned}
& \left(\zeta^{0}, \zeta^{0^{\prime}}, \zeta^{1}, \zeta^{1^{\prime}}, \ldots\right)
\end{aligned}
$$

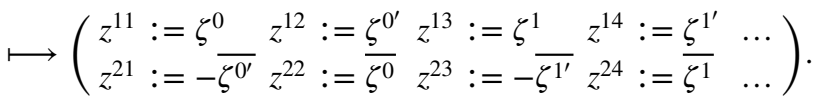

The coordinates on $\mathcal{W}=\left(\mathcal{U}_{0} / \mathbb{Z}_{2}\right) \times \mathrm{SL}_{2}(\mathbb{C}) \subset \mathcal{H}\left(\mathbb{M} P^{n}\right)^{\mathrm{qk}}$ which are defined by

$$
\left(x \equiv\left(z^{i a}\right), U=\left(u_{ \pm}^{i}\right)\right) \longmapsto\left(z^{ \pm a}=u_{i}^{ \pm} z^{i a},\left(u_{ \pm}^{i}\right)\right), \quad u_{i}^{ \pm}= \pm \epsilon_{i j} u_{\mp}^{j},
$$

are holomorphic adapted coordinates (as defined in Sect. 5.2.1; here, again $\left.\left(\epsilon_{j k}\right)=\left(\begin{array}{cc}0 & -1 \\ 1 & 0\end{array}\right)\right)$. Note that the coordinates $\left(z^{i a}, u_{ \pm}^{j}\right)$ are given in terms of the coordinates $\left(z^{ \pm a}, u_{ \pm}^{i}\right)$ by $z^{i a}=u_{+}^{i} z^{+a}+u_{-}^{i} z^{-a}$ and that $H_{0} \cdot z^{i a}=H_{++} \cdot z^{i a}=H_{--} \cdot z^{i a}=0$ for each $i=1,2$ and $1 \leq a \leq 2 n$.

For any quaternion $q=\zeta^{1}+\zeta^{2} \mathbf{j} \equiv\left(\zeta^{1}, \zeta^{2}\right)$, we denote by $\mathbb{M}(q)$ the matrix representing the linear map $R_{q}: \mathbb{U}=\mathbb{C}^{2} \rightarrow \mathbb{W}=\mathbb{C}^{2}$ determined by the right action $R_{q}$ of $q$. It is given by the $2 \times 2$ complex matrix

$$
\mathbb{M}(q)=\left(\begin{array}{cc}
\zeta^{1} & -\overline{\zeta^{2}} \\
\zeta^{2} & \overline{\zeta^{1}}
\end{array}\right) .
$$

Note that the standard complex conjugate $\overline{\mathbb{M}(q)}$ of the matrix associated with a quaternion $q=x_{0}+x_{1} \mathbf{i}+x_{2} \mathbf{j}+x_{3} \mathbf{k}$, is not equal to the matrix $\mathbb{M}\left(\bar{q}^{\mathbb{\sharp}}\right)$, associated with the conjugate quaternion $\bar{q}^{\sharp}:=x_{0}-x_{1} \mathbf{i}-x_{2} \mathbf{j}-x_{3} \mathbf{k}$. In fact, the proper relation is given by the conjugate transpose operator. Indeed,

$$
\begin{aligned}
\overline{\mathbb{M}\left(\zeta^{1}+\zeta^{2} \mathbf{j}\right)}= & \mathbb{M}\left(\overline{\zeta^{1}}+\overline{\zeta^{2}} \mathbf{j}\right), \quad \mathbb{M}\left(\zeta^{1}+\zeta^{2} \mathbf{j}\right)^{T}=\mathbb{M}\left(\zeta^{1}-\overline{\zeta^{2}} \mathbf{j}\right), \\
& \mathbb{M}\left(\zeta^{1}+\zeta^{2} \mathbf{j}\right)^{\dagger}=\mathbb{M}\left(\overline{\zeta^{1}}-\zeta^{2} \mathbf{j}\right)=\mathbb{M}\left(\overline{\left(\zeta^{1}+\zeta^{2} \mathbf{j}\right)}{ }^{\mathbb{A}}\right) .
\end{aligned}
$$

We now pick two integers $k, m \geq 1$ and consider an $(n+1)$-tuple of complex matrices $\underset{(a)}{\mathcal{A} \text {, }}$ $0 \leq a \leq n$, in $\mathbb{C}_{2 m \times 2 k}$, having the form

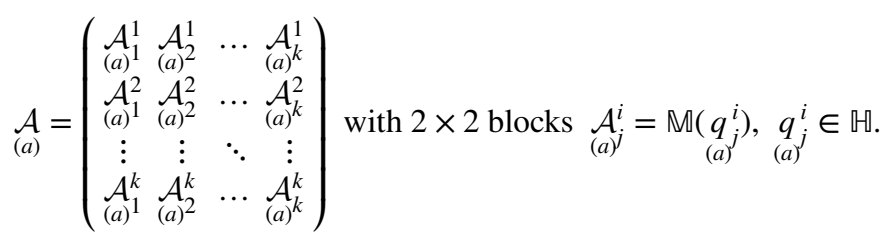

Note that by identifying each block $\mathbb{M}\left(\underset{(a)}{q_{j}^{i}}\right)$ with the corresponding quaternion $q_{j}^{i}$, the matrix $\mathcal{A}$ can be considered as an element of $\mathbb{U}_{m \times k}$ on which the $(\cdot)^{\dagger}$ operator acts as the $\mathbb{E}-$-conjugate transpose operator.

Finally, we consider the map $v: \mathcal{U}_{0} \rightarrow \mathbb{C}_{2 m \times 2 k}$ defined by 


$$
\begin{aligned}
& \left.v\right|_{\left(z^{j a}\right)} \\
& :=\underset{(0)}{\mathcal{A}}+\sum_{a=1}^{n} \underset{(a)}{\mathcal{A}}\left(\begin{array}{cclc}
\mathbb{M}\left(z^{1(2 a)}+z^{1(2 a+1)} \mathbf{j}\right) & 0 & \ldots & 0 \\
0 & \mathbb{M}\left(z^{1(2 a)}+z^{1(2 a+1)} \mathbf{j}\right) & \ldots & 0 \\
\vdots & \vdots & \ddots & \vdots \\
0 & 0 & \cdots & \mathbb{M}\left(z^{1(2 a)}+z^{1(2 a+1)} \mathbf{j}\right)
\end{array}\right)
\end{aligned}
$$

and we set $\lambda=-v^{\dagger}$. For this map $\lambda$ we have:

- At each point the corresponding map $\xi^{(\lambda)}$ is equal to a matrix with $2 \times 2$ blocks of the form $\mathbb{M}(q)$ for some quaternion $q$. It is therefore identifiable with a quaternionic matrix in $\mathbb{H}_{(k+m) \times m}$. This implies that for each $x \in \mathcal{U}_{0} \simeq \mathcal{W} \times\left\{I_{2}\right\}$, the matrix $\left.\xi^{(\lambda) \dagger}\left(\frac{\partial \xi^{(\lambda)}}{\partial z^{+a}}+\frac{\partial \xi^{(\lambda)}}{\partial z^{-a}}\right)\right|_{x}$ is an element in $\mathfrak{s t}_{2 m} \cap \mathfrak{g l} \mathfrak{l}_{m}(\mathbb{W})=\mathfrak{s p}_{m}$, i.e. $\lambda$ satisfies $(6.17)_{I}$ with $\mathfrak{g}^{0}=\mathfrak{s p}_{m}$.

- The matrix-valued function $\lambda_{\left(z^{i a}\right)}$ is independent of the coordinates $z^{ \pm 1}, z^{ \pm 2}$ and has trivial directional derivative along each of the vector fields $H_{A}, A \in\{0,++,--\}$. This implies that $\lambda$ trivially satisfies $(6.17)_{I I}$.

Due to this, if $v$ is such that conditions (6.17) $)_{I I I}$ and (6.17) $)_{I V}$ are also satisfied, we may conclude that $\lambda=-v^{\dagger}$ is the core of a prepotential. We now claim that these two conditions are satisfied if we impose that the $\mathcal{A}$ satisfy the additional algebraic conditions

$$
\underset{(a)(a)}{\mathcal{A} \mathcal{A}^{\dagger}}-\overline{(a)(a)}_{\mathcal{A}^{\dagger}}^{\mathbb{H}}=0, \quad \underset{(a)(b)}{\mathcal{A} \mathcal{A}^{\dagger}}=\underset{(b)(a)}{\mathcal{A} \mathcal{A}^{\dagger}}, \quad 0 \leq a, b \leq n .
$$

In fact, these conditions imply that the quaternionic entries of $\mathrm{I}_{2 k}+v^{\dagger} v$ have zero imaginary parts, that is they are real. Thus, for any $\alpha, \beta \in\{+,-\}$ and $2 \leq a, b \leq n+1$, we have

$$
\begin{aligned}
& \left(d v \wedge\left(\mathrm{I}_{2 k}+v^{\dagger} v\right)^{-1} d v^{\dagger}\right)\left(\frac{\partial}{\partial z^{\alpha a}}, \frac{\partial}{\partial z^{\beta b}}\right)=
\end{aligned}
$$

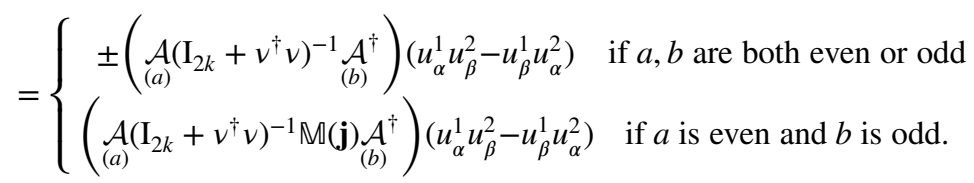

From this (6.17) $)_{I I I}$ and (6.17) $)_{I V}$ follow, as claimed.

We conclude that for any choice of the matrices as in (6.24), (6.25) and satisfying (6.26), the corresponding map $\lambda=-\nu^{\dagger}$ is the core of a prepotential of an instanton on $\hat{\mathcal{U}}_{0}=\pi\left(\mathcal{U}_{0}\right) \subset \mathbb{H} P^{n}$ with structure group $G^{o}=\mathrm{Sp}_{m}$. As mentioned above, instantons constructed in this way are precisely those constructed in [20, §3], where it was also proved that they are well defined over the entire $\mathbb{H} P^{n}$ and are characterised by certain topological properties. Further details of this class of instantons in the lowest dimensional case of $\mathbb{U} P^{1}=S^{4}$ can be found for instance in [3, Ch. II]. Explicit coordinate expressions for prepotentials for instantons on $S^{4}$ with structure group $G^{o}=\mathrm{Sp}_{1}$ were determined in [24].

As a final observation, we recall that by Theorem 5.4 any locally defined instanton on $M=\llbracket P^{n}$ is associated with an essentially unique local solution $\lambda$ to the system (6.17). Globally defined instantons thus correspond to sets of local solutions satisfying appropriate patching conditions on the overlapping domains. We believe that these patching conditions can be made fully explicit, at least in the case of a homogeneous qk manifold $M=H / K$ 
with $H$-invariant instantons. We are hopeful that this, together with a study of the local solution space of (6.17), will reveal new examples of instantons on compact homogeneous qk manifolds. We leave this task to future work.

Acknowledgements Open access funding provided by Projekt DEAL. CD thanks Hermann Nicolai and the Max-Planck-Institut für Gravitationsphysik (Albert-Einstein-Institut) for providing an excellent research environment. This research was partially supported by Ministero dell'Istruzione, Università e Ricerca in the framework of the project "Real and Complex Manifolds: Geometry, Topology and Harmonic Analysis" and by GNSAGA of INdAM.

Open Access This article is licensed under a Creative Commons Attribution 4.0 International License, which permits use, sharing, adaptation, distribution and reproduction in any medium or format, as long as you give appropriate credit to the original author(s) and the source, provide a link to the Creative Commons licence, and indicate if changes were made. The images or other third party material in this article are included in the article's Creative Commons licence, unless indicated otherwise in a credit line to the material. If material is not included in the article's Creative Commons licence and your intended use is not permitted by statutory regulation or exceeds the permitted use, you will need to obtain permission directly from the copyright holder. To view a copy of this licence, visit http://creativecommons.org/licenses/by/4.0/.

\section{References}

1. Alekseevsky, D.V., Cortes, V., Devchand, C.: Yang-Mills connections over manifolds with Grassmann structure. J. Math. Phys. 44, 6047-6074 (2003)

2. Alekseevsky, D.V., Marchiafava, S., Pontecorvo, M.: Compatible complex structures on almost quaternionic manifolds. Trans. Amer. Math. Soc. 351, 997-1014 (1999)

3. Atiyah, M.F.: Geometry of Yang-Mills Fields. Scuola Normale Superiore di Pisa, Pisa (1979)

4. Atiyah, M.F., Hitchin, N.J., Drinfel'd, V.G., Manin, Y.I.: Construction of instantons. Phys. Lett. A 65, 185-187 (1978)

5. Bergshoeff, E., Cucu, S., De Wit, T., Gheerardyn, J., Vandoren, S., Van Proeyen, A.: The map between conformal hypercomplex/hyperkähler and quaternionic(kähler) geometry. Comm. Math. Phys. 262, 411-457 (2006)

6. Corrigan, E., Devchand, C., Fairlie, D.B., Nuyts, J.: First-order equations for gauge fields in spaces of dimension greater than four. Nuclear Phys. B 214, 452-464 (1983)

7. Corrigan, E., Goddard, P., Kent, A.: Some comments on the ADHM construction in 4k dimensions. Comm. Math. Phys. 100, 1-13 (1985)

8. Devchand, C.: Oxidation of self-duality to 12 dimensions and beyond. Comm. Math. Phys. 329, 461$482(2014)$

9. Devchand, C., Lechtenfeld, O.: Extended self-dual Yang-Mills from the $N=2$ string. Nuclear Phys. B 516, 255-272 (1998)

10. Devchand, C., Leznov, A.N.: Bäcklund transformation for supersymmetric self-dual theories for semisimple gauge groups and a hierarchy of $A_{1}$ solutions. Comm. Math. Phys. 160, 551-561 (1994)

11. Devchand, C., Ogievetsky, V.: Interacting fields of arbitrary spin and $N>4$ supersymmetric self-dual Yang-Mills equations. Nuclear Phys. B 481, 188-214 (1996)

12. Devchand, C., Spiro, A.: On pseudo-hyperkähler prepotentials. J. Math. Phys. 57, 102501-102537 (2016)

13. Devchand, C., Pontecorvo, M., Spiro, A.: Instantons on hyperkähler manifolds. Ann. Mat. Pura Appl. (2019). https://doi.org/10.1007/s10231-019-00890-5

14. Galperin, A., Ivanov, E., Ogievetsky, V., Sokatchev, E.: Gauge field geometry from complex and harmonic Analyticities. I. Kähler and self-dual Yang-Mills cases. Ann. Physics 185, 1-21 (1988)

15. Galperin, A., Ivanov, E., Ogievetsky, O.: Harmonic space and quaternionic manifolds. Ann. Physics 230, 201-249 (1994)

16. Galperin, A.S., Ivanov, E.A., Ogievetsky, V.I., Sokatchev, E.S.: Harmonic Superspace. Cambridge University Press, Cambridge (2004)

17. Haydys, A.: HyperKähler and quaternionic Kähler manifolds with $S^{1}$-symmetries. J. Geom. Phys. 58, 293-306 (2008)

18. Hitchin, N.: On the hyperkähler/quaternion Kähler correspondence. Comm. Math. Phys. 324, 77-106 (2013) 
19. Leznov, A.N.: On the equivalence of four-dimensional self-duality equations to a continuous analogue of the problem of a principal chiral field. Teoret. Mat. Fiz. 73, 302-307 (1987) (English translation: Theoret. and Math. Phys. 73, 1233-1236 (1987))

20. Mamone Capria, M., Salamon, S.M.: Yang-Mills fields on quaternionic spaces. Nonlinearity 1, 517530 (1988)

21. Narasimhan, M.S., Ramanan, S.: Existence of universal connection. Amer. J. Math. 83, 563-572 (1961)

22. Narasimhan, M.S., Ramanan, S.: Existence of universal connections. II. Amer. J. Math. 85, 223-231 (1963)

23. Nitta, T.: Vector bundles over quaternionic Kähler manifolds. Tohoku Math. J. 40, 425-440 (1988)

24. Ogievetsky, O.: Harmonic representatives of instantons and self-dual monopoles. In: Group Theoretical Methods in Physics (Varna, 1987), Lecture Notes in Phys., vol. 313, pp. 548-554. Springer, Berlin (1988)

25. Pedersen, H., Poon, Y.S., Swann, A.F.: Hypercomplex structures associated to quaternionic manifolds. Differential Geom. Appl. 9, 273-292 (1998)

26. Salamon, S.M.: Quaternionic Kähler manifolds. Invent. Math. 67, 143-171 (1982)

27. Salamon, S.M.: Differential geometry of quaternionic manifolds. Ann. scient. Éc. Norm. sup., $4^{a}$ sèrie 19, 31-55 (1986)

28. Siegel, W.: $N=2$ (4) string theory is self-dual $N=4$ Yang-Mills theory. Phys. Rev. D (3) 46, R3235R3238 (1992)

29. Swann, A.: HyperKähler and quaternionic Kähler geometry. Math. Ann. 289, 421-450 (1991)

30. Tian, G.: Gauge theory and calibrated geometry, I. Ann. of Math. 151, 193-208 (2000)

31. Uhlenbeck, K.K.: Equations of Gauge Theories, notes by L. Fredrickson, lecture at Temple University, Philadelphia. https://web.stanford.edu/ ljfred4/Attachments/TempleLectures.pdf (2012)

32. Ward, R.S.: Completely solvable gauge-field equations in dimension greater than four. Nuclear Phys. B 236, 381-396 (1984)

Publisher's Note Springer Nature remains neutral with regard to jurisdictional claims in published maps and institutional affiliations. 